\title{
On a Boundary Value Problem for the Biharmonic Equation with Multiple Involutions
}

\author{
Batirkhan Turmetov $^{1, *,+(\mathbb{D}) \text {, Valery Karachik }}{ }^{2,+}(\mathbb{D})$ and Moldir Muratbekova ${ }^{1,+}+\mathbb{D}$ \\ 1 Department of Mathematics, Khoja Akhmet Yassawi International Kazakh-Turkish University, \\ Turkistan 161200, Kazakhstan; moldir_1983@mail.ru \\ 2 Department of Mathematical Analysis, South Ural State University (NRU), 454080 Chelyabinsk, Russia; \\ karachik@susu.ru \\ * Correspondence: batirkhan.turmetov@ayu.edu.kz \\ + These authors contributed equally to this work.
}

Citation: Turmetov, B.; Karachik, V.; Muratbekova, M. On a Boundary Value Problem for the Biharmonic Equation with Multiple Involutions Mathematics 2021, 9, 2020. https:/ / doi.org/10.3390/math9172020

Academic Editors: F. Adrián F. Tojo and Marlène Frigon

Received: 29 July 2021

Accepted: 18 August 2021

Published: 24 August 2021

Publisher's Note: MDPI stays neutral with regard to jurisdictional claims in published maps and institutional affiliations.

Copyright: (C) 2021 by the authors. Licensee MDPI, Basel, Switzerland. This article is an open access article distributed under the terms and conditions of the Creative Commons Attribution (CC BY) license (https:// creativecommons.org/licenses/by/ $4.0 /)$.

\begin{abstract}
A nonlocal analogue of the biharmonic operator with involution-type transformations was considered. For the corresponding biharmonic equation with involution, we investigated the solvability of boundary value problems with a fractional-order boundary operator having a derivative of the Hadamard-type. First, transformations of the involution type were considered. The properties of the matrices of these transformations were investigated. As applications of the considered transformations, the questions about the solvability of a boundary value problem for a nonlocal biharmonic equation were studied. Modified Hadamard derivatives were considered as the boundary operator. The considered problems covered the Dirichlet and Neumann-type boundary conditions. Theorems on the existence and uniqueness of solutions to the studied problems were proven.
\end{abstract}

Keywords: boundary value problems; biharmonic equation; multiple involutions; fractional derivative; Hadamard operator

\section{Introduction}

The concept of a nonlocal operator and the related concepts of a nonlocal differential equation appeared in the theory of differential equations not long ago. For example, in [1], the authors considered equations containing fractional derivatives of the desired function and equations with deviating arguments, in other words, equations that include an unknown function and its derivatives, generally speaking, for different values of arguments. Such equations are called nonlocal differential equations. Among nonlocal differential equations, a special place is occupied by equations in which the deviation of the arguments has an involutive character. Mapping $S$ is usually called an involution if $S^{2}(x)=S(S(x))=x$. The theory of equations with involutively transformed arguments and their applications were described in detail in the monographs [2-4]. By now, for differential equations with various types of involution, the well-posedness of boundary and initial-boundary value problems, the qualitative properties of solutions, and spectral issues have been studied quite well [5-16]. In addition, for classical equations, one can study nonlocal boundary value problems of the Bitsadze-Samarskii-type, in which the values of the sought function $u(x)$ at the boundary of the domain are related to the values of $u(S x)$ [17-19]. Note that the questions about the solvability of the main problems for the nonlocal Poisson equation were studied in $[20,21]$. Note also that boundary value problems with fractional-order boundary operators for elliptic equations were studied in [22-30].

Applications of the boundary value problems with boundary operators of fractionalorder for elliptic equations were given in [31-34].

Let us formulate the problem that is studied in this work. Let $\Omega$ be the unit ball in $\mathbb{R}^{l}, l \geq 2$, and $\partial \Omega$ be the unit sphere. Let also $u(x)$ be a smooth function in the domain 
$\Omega, \mu \geq 0, \alpha \in(m-1, m], m=1,2, \ldots, r=|x|=\sqrt{x_{1}^{2}+\ldots+x_{n}^{2}}, \theta=x / r, x \in \Omega$, and $\delta=r \frac{d}{d r}$ be the Dirac operator, where $r \frac{d}{d r}=\sum_{j=1}^{l} x_{j} \frac{\partial}{\partial x_{j}}$. Consider the modified Hadamard integrodifferential operators ([35], p. 116):

$$
\begin{aligned}
& J_{\mu}^{\alpha}[u](x)=\left\{\begin{array}{c}
u(x), \alpha=0, \\
\frac{1}{\Gamma(\alpha)} \int_{0}^{1}\left(\ln \frac{1}{\tau}\right)^{\alpha-1} \tau^{\mu-1} u(\tau x) d \tau, \alpha>0
\end{array},\right. \\
& D_{\mu}^{\alpha}[u](x)=r^{-\mu} J^{m-\alpha}\left[\delta^{m}\left[\tau^{\mu} \cdot u\right]\right](x), m-1<\alpha \leq m .
\end{aligned}
$$

Let $S_{1}, \ldots, S_{n}$ be a set of real symmetric commutative matrices $S_{i} S_{j}=S_{j} S_{i}$ such that $S_{i}^{2}=I$. Note that, since any transform $S_{i}$ is isometric, then if $x \in \Omega$ or $x \in \partial \Omega$, then $S x \in \Omega$ or $S x \in \partial \Omega$, respectively. For example, matrix $S_{i}$ can be a matrix of the following linear mapping $S_{i} x=\left(x_{1}, \ldots, x_{i-1},-x_{i}, x_{i+1}, \ldots, x_{l}\right)$.

Let $a_{0}, a_{1}, a_{2}, a_{3}, \ldots, a_{2^{n}-1}$ be a set of real numbers. If we write the summation index $i$ in the binary number system $\left(i_{n} \ldots i_{1}\right)_{2} \equiv i$, where $i_{k}=0,1$ for $k=1, \ldots, n$, then the coefficients can be written as $a_{(0 \ldots . .00)_{2}}, a_{(0 \ldots 01)_{2}}, a_{(0 \ldots 10)_{2}}, a_{(0 \ldots 11)_{2}, \ldots, a_{(1 \ldots 1)_{2}}}$.

Let us introduce the following nonlocal analogue of the biharmonic operator:

$$
L_{n} u(x) \equiv \sum_{i=0}^{2^{n}-1} a_{i} \Delta^{2} u\left(S_{n}^{i_{n}} \ldots S_{1}^{i_{1}} x\right) .
$$

Consider the following boundary value problem in the domain $\Omega$.

Problem H. Let $\mu \geq 0,0 \leq \alpha \leq 1$. Find the function $u(x) \in C^{4}(\Omega) \cap C(\bar{\Omega})$, for which functions $D_{\mu}^{\alpha}[u](x), D_{\mu}^{\alpha+1}[u](x)$ are continuous in $\bar{\Omega}$ and satisfy the conditions:

$$
\begin{gathered}
L_{n} u(x)=f(x), x \in \Omega, \\
\left.D_{\mu}^{\alpha}[u](x)\right|_{\partial \Omega}=g_{0}(x), x \in \partial \Omega, \\
\left.D_{\mu}^{\alpha+1}[u](x)\right|_{\partial \Omega}=g_{1}(x), x \in \partial \Omega .
\end{gathered}
$$

Remark 1. Note that in the case $a_{0}=1, a_{k}=0, k=1,2, \ldots, 2^{n}-1$, Equation (1) coincides with the classical inhomogeneous biharmonic equation.

Remark 2. Note also that for $x \in \partial \Omega$, the following equalities hold:

$$
\begin{gathered}
\left.\delta[u](x)\right|_{\partial \Omega}=\left.r \frac{d u(x)}{d r}\right|_{\partial \Omega}=\left.\frac{\partial u(x)}{\partial v}\right|_{\partial \Omega}{ }^{\prime} \\
\left.\frac{\partial^{2} u(x)}{\partial v^{2}}\right|_{\partial \Omega}=\left.r \frac{d}{d r}\left(r \frac{d}{d r}-1\right) u(x)\right|_{\partial \Omega}=\left.r^{2} \frac{d^{2} u(x)}{d r^{2}}\right|_{\partial \Omega},
\end{gathered}
$$

where $v$ is the outer normal to the sphere $\partial \Omega$. Then, for $\alpha=1$, we obtain:

$$
\begin{aligned}
D_{\mu}^{1}[u](x) & =r^{-\mu} J^{0}\left[\delta\left[\tau^{\mu} \cdot u\right]\right](x)=r^{-\mu} r \frac{d}{d r}\left[r^{\mu} u(x)\right]=r \frac{d}{d r} u(x)+\mu u(x), \\
D_{\mu}^{2}[u](x) & =r^{-\mu}\left(r \frac{d}{d r}\right)^{2}\left[r^{\mu} u(x)\right]=r^{1-\mu} \frac{d}{d r}\left(r^{\mu+1} \frac{d}{d r} u(x)+\mu r^{\mu} u(x)\right) \\
= & r^{2} \frac{d^{2} u(x)}{d r^{2}}+(\mu+1) r \frac{d}{d r} u(x)+\mu r \frac{d}{d r} u(x)+\mu^{2} u(x)
\end{aligned}
$$




$$
=r^{2} \frac{d^{2} u(x)}{d r^{2}}+(2 \mu+1) r \frac{d}{d r} u(x)+\mu^{2} u(x) .
$$

Thus, if $\mu=0$, then in the case $\alpha=0$ in the problem $H$, we obtain the Dirichlet boundary conditions:

$$
u(x)=g_{0}(x), \quad \frac{\partial u(x)}{\partial v}=g_{1}(x), x \in \partial \Omega,
$$

and in the case $\alpha=1$, boundary conditions of the Neumann-type [36,37]:

$$
\frac{\partial u(x)}{\partial v}=g_{0}(x), \quad \frac{\partial^{2} u(x)}{\partial v^{2}}+\frac{\partial u(x)}{\partial v}=g_{1}(x), x \in \partial \Omega .
$$

Moreover, in the case $\alpha=1$ and $\mu>0$, the boundary conditions of the problem $H$ are written as:

$$
\frac{\partial u(x)}{\partial v}+\mu u(x)=g_{0}(x), \quad \frac{\partial^{2} u(x)}{\partial v^{2}}+(2 \mu+1) \frac{\partial u(x)}{\partial v}+\mu^{2} u(x)=g_{1}(x), x \in \partial \Omega,
$$

i.e., we obtain a generalization of the third boundary value problem [38].

The article is organized as follows. In Section 1, the main problem is formulated. In Section 2, the properties of matrices of involutive transformations are considered. Section 3 contains well-known statements about the properties of Hadamard integrodifferential operators. In Section 4, the solvability of the Dirichlet problem (case $\alpha=0$ ) is studied, and in Section 5, the necessary and sufficient conditions for the solvability of Neumann-type boundary value problems (case $\mu=0, \alpha=1$ ) are found. In Section 6, for the general case, the main boundary value problem is studied.

\section{Auxiliary Statements}

To study the problem (1)-(3), we need some auxiliary statements. Let us introduce the function:

$$
v(x)=\sum_{i \equiv\left(i_{n} \ldots i_{1}\right)_{2}=0}^{(1 \ldots 1)_{2}} a_{i} u\left(S_{n}^{i_{n}} \ldots S_{1}^{i_{1}} x\right),
$$

where the summation is taken in ascending order with respect to the index $i$. From this equality, it is easy to conclude that functions of the form $v\left(S_{n}^{j_{n}} \ldots S_{1}^{j_{1}} x\right)$, where $j=0, \ldots, 2^{n}-1$, can be linearly expressed in terms of functions $u\left(S_{n}^{i_{n}} \ldots S_{1}^{i_{1}} x\right)$. If we consider the following vectors of order $2^{n}$ :

$$
\begin{aligned}
& U(x)=\left(u(x), \ldots, u\left(S_{n}^{i_{n}} \ldots S_{1}^{i_{1}} x\right), \ldots, u\left(S_{n}^{1} \ldots S_{1}^{1} x\right)\right)^{T}, \\
& V(x)=\left(v(x), \ldots, v\left(S_{n}^{j_{n}} \ldots S_{1}^{j_{1}} x\right), \ldots, v\left(S_{n}^{1} \ldots S_{1}^{1} x\right)\right)^{T},
\end{aligned}
$$

then this dependence can be expressed in the matrix form:

$$
V(x)=A_{n} U(x),
$$

where $A_{n}=\left(a_{i, j}\right)_{i, j=0, \ldots, 2^{n}-1}$ is the matrix of order $2^{n} \times 2^{n}$.

Theorem 1. The matrix $A_{n}$ from the equality (5) can be represented in the form:

$$
A_{n}=\left(a_{i, j}\right)_{i, j=0, \ldots, 2^{n}-1}=\left(a_{i \oplus j}\right)_{i, j=0, \ldots, 2^{n}-1^{\prime}}
$$

where the operation in the subscript of the matrix coefficients is understood in the following sense $i \oplus j \equiv(i)_{2} \oplus(j)_{2}=\left(\left(i_{n}+j_{n} \bmod 2\right) \ldots\left(i_{1}+j_{1} \bmod 2\right)\right)_{2}$, where $(i)_{2}=\left(i_{n} \ldots i_{1}\right)_{2}$ is a 
representation of the index in the binary number system. The linear combination of matrices of the form of (6) is a matrix of the form of (6).

Proof. Let $n=1$, then we have:

$$
A_{1}=\left(\begin{array}{ll}
a_{0 \oplus 0} & a_{0 \oplus 1} \\
a_{1 \oplus 0} & a_{1 \oplus 1}
\end{array}\right)=\left(\begin{array}{ll}
a_{0} & a_{1} \\
a_{1} & a_{0}
\end{array}\right)
$$

and if $n=2$, then we obtain:

$$
A_{2}=\left(\begin{array}{llll}
a_{(00)_{2} \oplus(00)_{2}} & a_{(00)_{2} \oplus(01)_{2}} & a_{(00)_{2} \oplus(10)_{2}} & a_{(00)_{2} \oplus(11)_{2}} \\
a_{(01)_{2} \oplus(00)_{2}} & a_{(01)_{2} \oplus(01)_{2}} & a_{(01)_{2} \oplus(10)_{2}} & a_{(01)_{2} \oplus(11)_{2}} \\
a_{(10)_{2} \oplus(00)_{2}} & a_{(10)_{2} \oplus(01)_{2}} & a_{(10)_{2} \oplus(10)_{2}} & a_{(10)_{2} \oplus(11)_{2}} \\
a_{(11)_{2} \oplus(00)_{2}} & a_{(11)_{2} \oplus(01)_{2}} & a_{(11)_{2} \oplus(10)_{2}} & a_{(11)_{2} \oplus(11)_{2}}
\end{array}\right)=\left(\begin{array}{cccc}
a_{0} & a_{1} & a_{2} & a_{3} \\
a_{1} & a_{0} & a_{3} & a_{2} \\
a_{2} & a_{3} & a_{0} & a_{1} \\
a_{3} & a_{2} & a_{1} & a_{0}
\end{array}\right)
$$

Consider the function $v\left(S_{n}^{i_{n}} \ldots S_{1}^{i_{1}} x\right)$ whose coefficients at $u\left(S_{n}^{j_{n}} \ldots S_{1}^{j_{1}}\right)$ make up the $i \equiv\left(i_{n} \ldots i_{1}\right)_{2}$ th row of the matrix $A_{n}$ :

$$
\begin{aligned}
v\left(S_{n}^{i_{n}} \ldots S_{1}^{i_{1}} x\right)=\sum_{j \equiv\left(j_{n} \ldots j_{1}\right)_{2}=0}^{2^{n}-1=(1 \ldots 1)_{2}} a_{\left(j_{n} \ldots j_{1}\right)_{2}} u\left(S_{n}^{j_{n}} \ldots S_{1}^{j_{1}} S_{n}^{i_{n}} \ldots S_{1}^{i_{1}} x\right) \\
=\sum_{j \equiv\left(j_{n} \ldots j_{1}\right)_{2}=0}^{(1 \ldots 1)_{2}} a_{\left(j_{n} \ldots j_{1}\right)_{2}} u\left(S_{n}^{j_{n}+i_{n} \bmod 2} \ldots S_{1}^{j_{1}+i_{1} \bmod 2} x\right) .
\end{aligned}
$$

Here, the following properties $S_{j}^{2} x=x$ and $S_{j} S_{i} x=S_{i} S_{j} x$ of matrices $S_{1}, \ldots, S_{n}$ are taken into account. Let us replace the index $i \oplus j=l$. Then, $l \oplus i=i \oplus j \oplus i=j$, and the correspondence $j \sim l$ is one-to-one. Replacement $j \rightarrow l$ of the index changes only the order of summation in the sum (7). For example, if $i=1$, then the sequence $j: 0,1,2,3,4,5, \ldots$ goes to $l=1 \oplus j: 1,0,3,2,5,4, \ldots$. After replacing the index, we obtain:

$$
v\left(S_{n}^{i_{n}} \ldots S_{1}^{i_{1}} x\right)=\sum_{l=0}^{(1 \ldots 1)_{2}} a_{\left(i_{n}+l_{n} \bmod 2 \ldots i_{1}+l_{1} \bmod 2\right)_{2}} u\left(S_{n}^{l_{n}} \ldots S_{1}^{l_{1}} x\right),
$$

whence $a_{i, l}=a_{\left(i_{n}+l_{n} \bmod 2 \ldots i_{1}+l_{1} \bmod 2\right)_{2}}=a_{i \oplus l}$, which proves (6).

It is clear that if $\alpha, \beta$ are constants, then:

$$
\alpha\left(a_{i \oplus j}\right)_{i, j=0, \ldots, 2^{n}-1}+\beta\left(b_{i \oplus j}\right)_{i, j=0, \ldots, 2^{n}-1}=\left(\alpha a_{i \oplus j}+\beta b_{i \oplus j}\right)_{i, j=0, \ldots, 2^{n}-1} .
$$

The theorem is proven.

Corollary 1. The matrix $A_{n}$ is uniquely determined by its first row $\left(a_{0}, a_{1}, \ldots, a_{2^{n}-1}\right)$.

Indeed, the $i$ th row of the matrix $A_{n}$ can be written through its first row in the form $\left(a_{i \oplus 0}, a_{i \oplus 1}, \ldots, a_{i \oplus\left(2^{n}-1\right)}\right)$.

This property of the matrix $A_{n}$ we denote by the equality $A_{n} \equiv A_{n}\left(a_{0}, \ldots, a_{2^{n}-1}\right)$.

Corollary 2. The matrix $A_{n}$ has the symmetry property:

$$
\left(a_{i, j}\right)_{i, j=0, \ldots, 2^{n}-1}=\left(a_{j, i}\right)_{i, j=0, \ldots, 2^{n}-1}
$$

and can be written as:

$$
A_{n}=\left(\begin{array}{cc}
A_{n-1}\left(a_{0}, \ldots, a_{2^{n-1}-1}\right) & A_{n-1}\left(a_{2^{n-1}}, \ldots, a_{2^{n}-1}\right) \\
A_{n-1}\left(a_{2^{n-1}}, \ldots, a_{2^{n}-1}\right) & A_{n-1}\left(a_{0}, \ldots, a_{2^{n-1}-1}\right)
\end{array}\right)
$$


or more generally in the form of a block matrix $A_{n-m}$ consisting of matrices $A_{m}$ :

$$
A_{n}=A_{n-m}\left(A_{m}^{(0 . \ldots)_{2}}, \ldots, A_{m}^{\left(k_{n} \ldots k_{m+1}\right)_{2}}, \ldots, A_{m}^{(1 \ldots 1)_{2}}\right),
$$

where $A_{m}^{\left(k_{n} \ldots k_{m+1}\right)_{2}}\left(a_{\left(k_{n} \ldots k_{m+1} 0 \ldots 0\right)_{2}}, \ldots, a_{\left(k_{n} \ldots k_{m+1} 1 \ldots 1\right)_{2}}\right)$ is a matrix of the form of $(6)$ of order $2^{m}$.

Proof. Indeed, since the binary operation $i \oplus j$ is commutative:

$$
i \oplus j=\left(i_{n}+j_{n} \bmod 2 \ldots i_{1}+j_{1} \bmod 2\right)_{2}=\left(j_{n}+i_{n} \bmod 2 \ldots j_{1}+i_{1} \bmod 2\right)_{2}=j \oplus i,
$$

then the property (8) holds:

$$
\left(a_{i, j}\right)_{i, j=0, \ldots, 2^{n}-1}=\left(a_{i \oplus j}\right)_{i, j=0, \ldots, 2^{n}-1}=\left(a_{j \oplus i}\right)_{i, j=0, \ldots, 2^{n}-1}=\left(a_{j, i}\right)_{i, j=0, \ldots, 2^{n}-1} .
$$

Further, it is easy to see the validity of the equalities:

$$
\left(a_{\left(0 i_{n-1} \ldots i_{1}\right)_{2} \oplus\left(0 j_{n-1} \ldots j_{1}\right)_{2}}\right)_{i, j=0, \ldots, 2^{2-1}-1}=\left(a_{\left(1 i_{n-1} \ldots i_{1}\right)_{2} \oplus\left(1 j_{n-1} \ldots j_{1}\right)_{2}}\right)_{i, j=0, \ldots, 2^{n-1}-1}
$$

and:

$$
\left(a_{\left(0 i_{n-1} \ldots i_{1}\right)_{2} \oplus\left(1 j_{n-1} \ldots j_{1}\right)_{2}}\right)_{i, j=0, \ldots, 2^{n-1}-1}=\left(a_{\left(1 i_{n-1} \ldots i_{1}\right)_{2} \oplus\left(0 j_{n-1} \ldots j_{1}\right)_{2}}\right)_{i, j=0, \ldots, 2^{n-1}-1^{\prime}}
$$

from which the property (9) follows. Indeed, if we divide the matrix $A_{n}$ into four equalin-size square blocks and consider the lower right block, then its indices are located in the range $(10 \ldots 0)_{2} \leq i, j \leq(11 \ldots 1)_{2}$, which means that this block, by virtue of (11), has the form:

$$
\begin{aligned}
\left(a_{\left(1 i_{n-1} \ldots i_{1}\right)_{2} \oplus\left(1 j_{n-1} \ldots j_{1}\right)_{2}}\right. & )_{i, j=0, \ldots, 2^{n-1}-1} \\
& =\left(a_{\left(0 i_{n-1} \ldots i_{1}\right)_{2} \oplus\left(0 j_{n-1} \ldots j_{1}\right)_{2}}\right)_{i, j=0, \ldots, 2^{n-1}-1}=A_{n-1}\left(a_{0}, \ldots, a_{2^{n-1}-1}\right),
\end{aligned}
$$

i.e., the diagonal blocks of the matrix $A_{n}$ are of the form $A_{n-1}\left(a_{0}, \ldots, a_{2^{n-1}-1}\right)$.

Similarly, the top right block of $A_{n}$ has the indices in the range $(00 \ldots 0)_{2} \leq i \leq$ $(01 \ldots 1)_{2},(10 \ldots 0)_{2} \leq j \leq(11 \ldots 1)_{2}$, which means this block has the form:

$$
\left(a_{\left(0 i_{n-1} \ldots i_{1}\right)_{2} \oplus\left(1 j_{n-1} \ldots j_{1}\right)_{2}}\right)_{i, j=0, \ldots, 2^{n-1}-1}=A_{n-1}\left(a_{2^{n-1}}, \ldots, a_{2^{n}-1}\right) .
$$

By the equality (12), the lower left block of $A_{n}$ has the form:

$$
\begin{aligned}
& \left(a_{\left(1 i_{n-1} \ldots i_{1}\right)_{2} \oplus\left(0 j_{n-1} \ldots j_{1}\right)_{2}}\right)_{i, j=0, \ldots, 2^{n-1}-1} \\
& \quad=\left(a_{\left(0 i_{n-1} \ldots i_{1}\right)_{2} \oplus\left(1 j_{n-1} \ldots j_{1}\right)_{2}}\right)_{i, j=0, \ldots, 2^{n-1}-1}=A_{n-1}\left(a_{2^{n-1}}, \ldots, a_{2^{n}-1}\right) .
\end{aligned}
$$

Equality (9) is proven. Now, consider a block matrix of the form:

$$
A_{n-m}\left(A_{m}^{(0 \ldots 0)_{2}}, \ldots, A_{m}^{\left(k_{n} \ldots k_{m+1}\right)_{2}}, \ldots, A_{m}^{(1 \ldots 1)_{2}}\right)=\left(A_{m}^{\left(i_{n} \ldots i_{m+1}\right)_{2} \oplus\left(j_{n} \ldots j_{m+1}\right)_{2}}\right)_{i, j=0, \ldots, 2^{n-m}-1} .
$$

The elements of its block matrix with the number $\left(k_{n} \ldots k_{m+1}\right)_{2}$ can be written as:

$$
A_{m}^{\left(k_{n} \ldots k_{m+1}\right)_{2}}\left(a_{\left(k_{n} \ldots k_{m+1} 0 \ldots 0\right)_{2}} \ldots, a_{\left(k_{n} \ldots k_{m+1} 1 \ldots 1\right)_{2}}\right)=\left(a_{\left(k_{n} \ldots k_{m+1}\left(i_{m} \ldots i_{1}\right)_{2} \oplus\left(j_{m} \ldots j_{1}\right)_{2}\right)}\right)_{i, j=0, \ldots, 2^{m}-1} .
$$


Consider the element $a_{i, j}$ of the block matrix:

$$
A_{n-m}\left(A_{m}^{(0 \ldots 0)_{2}}, \ldots, A_{m}^{\left(k_{n} \ldots k_{m+1}\right)_{2}}, \ldots, A_{m}^{(1 \ldots 1)_{2}}\right) .
$$

It is located in the block with coordinates $\left(i_{n} \ldots i_{m+1}\right)_{2},\left(j_{n} \ldots j_{m+1}\right)_{2}$, and this means it is in the block $A_{m}^{\left(i_{n} \ldots i_{m+1}\right)_{2} \oplus\left(j_{n} \ldots j_{m+1}\right)_{2}}$ and, therefore, has the form:

$$
a_{i, j}=a_{\left(\left(i_{n} \ldots i_{m+1}\right)_{2} \oplus\left(j_{n} \ldots j_{m+1}\right)_{2}\left(i_{m} \ldots i_{1}\right)_{2} \oplus\left(j_{m} \ldots j_{1}\right)_{2}\right)}=a_{i \oplus j} .
$$

This coincides with Formula (6). The corollary is proven.

Theorem 2. Multiplication of matrices of the form of (6) is commutative. The product of matrices of the form of (6) is again a matrix of the form of (6).

Proof. Let us prove this property by induction on $n$. For $n=1$, it is obviously true that:

$$
\begin{aligned}
& A_{1} B_{1}=\left(\begin{array}{ll}
a_{0} & a_{1} \\
a_{1} & a_{0}
\end{array}\right)\left(\begin{array}{ll}
b_{0} & b_{1} \\
b_{1} & b_{0}
\end{array}\right)=\left(\begin{array}{ll}
a_{0} b_{0}+a_{1} b_{1} & a_{0} b_{1}+a_{1} b_{0} \\
a_{1} b_{0}+a_{0} b_{1} & a_{1} b_{1}+a_{0} b_{0}
\end{array}\right) \\
&=\left(\begin{array}{ll}
b_{0} a_{0}+b_{1} a_{1} & b_{0} a_{1}+b_{1} a_{0} \\
b_{1} a_{0}+b_{0} a_{1} & b_{1} a_{1}+b_{0} a_{0}
\end{array}\right)=B_{1} A_{1} .
\end{aligned}
$$

Assuming that the multiplication of matrices $A_{n-1}$ and $B_{n-1}$ of order $n-1$ is commutative, we prove that the multiplication of matrices $A_{n}$ and $B_{n}$ of order $n$ is also commutative. Denote $A_{n-1}=A_{n-1}\left(a_{0}, \ldots, a_{2^{n-1}-1}\right), \hat{A}_{n-1}=A_{n-1}\left(a_{2^{n-1}}, \ldots, a_{2^{n}-1}\right)$. By the property (9):

$$
\begin{gathered}
A_{n} B_{n}=\left(\begin{array}{ll}
A_{n-1} & \hat{A}_{n-1} \\
\hat{A}_{n-1} & A_{n-1}
\end{array}\right)\left(\begin{array}{ll}
B_{n-1} & \hat{B}_{n-1} \\
\hat{B}_{n-1} & B_{n-1}
\end{array}\right)=\left(\begin{array}{ll}
A_{n-1} B_{n-1}+\hat{A}_{n-1} \hat{B}_{n-1} & A_{n-1} \hat{B}_{n-1}+\hat{A}_{n-1} B_{n-1} \\
\hat{A}_{n-1} B_{n-1}+A_{n-1} \hat{B}_{n-1} & \hat{A}_{n-1} \hat{B}_{n-1}+A_{n-1} B_{n-1}
\end{array}\right) \\
=\left(\begin{array}{ll}
B_{n-1} A_{n-1}+\hat{B}_{n-1} \hat{A}_{n-1} & B_{n-1} \hat{A}_{n-1}+\hat{B}_{n-1} A_{n-1} \\
\hat{B}_{n-1} A_{n-1}+B_{n-1} \hat{A}_{n-1} & \hat{B}_{n-1} \hat{A}_{n-1}+B_{n-1} A_{n-1}
\end{array}\right)=B_{n} A_{n} .
\end{gathered}
$$

The induction step is proven, and therefore, matrices of the form $A_{n}\left(a_{0}, \ldots, a_{2^{n}-1}\right)$ are commutative. It is not hard to see that:

$$
A B=\left(a_{i \oplus j}\right)_{i, j=0, \ldots, 2^{n}-1}\left(b_{i \oplus j}\right)_{i, j=0, \ldots, 2^{n}-1}=\left(\sum_{k=0}^{2^{n}-1} a_{i \oplus k} b_{k \oplus j}\right)_{i, j=0, \ldots, 2^{n}-1} .
$$

In the sum from the formula above, let us change the index $k \rightarrow l$, as in Theorem 1 , according to the equality $i \oplus k=l$. Then, $l \oplus i=i \oplus k \oplus i=i \oplus i \oplus k=k$, and this means that the correspondence $k \sim l$ is one-to-one. Replacement of the index $k \rightarrow l$ changes only the order of summation in the sum. By virtue of the associatively of the operation $\oplus$, we have:

$$
A B=\left(\sum_{l=0}^{2^{n}-1} a_{l} b_{(l \oplus i) \oplus j}\right)_{i, j=0, \ldots, 2^{n}-1}=\left(\sum_{l=0}^{2^{n}-1} a_{l} b_{l \oplus(i \oplus j)}\right)_{i, j=0, \ldots, 2^{n}-1} .
$$

The first row of the matrix $A B$ is:

$$
(A B)_{i=0}=\left(\sum_{k=0}^{2^{n}-1} a_{k} b_{k \oplus j}\right)_{j=0, \ldots, 2^{n}-1},
$$


and hence, the matrix $C$ of the form of (6), constructed by the first row of $A B$, is written in the form coinciding with $A B$ :

$$
C \equiv\left(\sum_{k=0}^{2^{n}-1} a_{k} b_{k \oplus(i \oplus j)}\right)_{j=0, \ldots, 2^{n}-1}=A B .
$$

The theorem is proven.

Theorem 3. The eigenvectors of the matrix $A_{n}\left(a_{0}, \ldots, a_{2^{n}-1}\right)$ can be chosen in the form:

$$
\mathbf{a}_{n}^{k}=\left(\mathbf{a}_{n-1}^{k}, \pm \mathbf{a}_{n-1}^{k}\right)^{T}, k=0, \ldots, 2^{n-1}-1
$$

where $\mathbf{a}_{n-1}^{k}$ is the eigenvector of the matrix $A_{n-1}\left(a_{0}, \ldots, a_{2^{n-1}-1}\right), k=0, \ldots, 2^{n-1}-1$; for $n=1$, we have $\mathbf{a}_{1}^{0}=(1,1)^{T}, \mathbf{a}_{1}^{1}=(1,-1)^{T}$. The eigenvectors of the matrix $A_{n}$ are orthogonal. The eigenvalues of the matrix $A_{n}$ are of the form:

$$
\mu_{n}^{k, \pm}=\mu_{n-1}^{k} \pm \hat{\mu}_{n-1}^{k}, k=0, \ldots, 2^{n-1}-1,
$$

where $\mu_{n-1}^{k}$ and $\hat{\mu}_{n-1}^{k}$ are eigenvalues of the matrices:

$$
A_{n-1}\left(a_{0}, \ldots, a_{2^{n-1}-1}\right) \text { and } \hat{A}_{n-1}=A_{n-1}\left(a_{2^{n-1}}, \ldots, a_{2^{n}-1}\right)
$$

corresponding to the eigenvector $\mathbf{a}_{n-1}^{k}$, respectively; $\mu_{1}^{0}=a_{0}+a_{1}, \mu_{1}^{1}=a_{0}-a_{1}$.

Proof. Let us carry out the proof by induction on $n$. Suppose that the eigenvectors of the matrix $A_{n}\left(a_{0}, \ldots, a_{2^{n}-1}\right)$ are independent on numbers $a_{0}, \ldots, a_{2^{n}-1}$. For $n=1$, it is obvious that the eigenvectors of the matrix $A_{1}\left(a_{0}, a_{1}\right)$ can be chosen in the form $\mathbf{a}_{1}^{+}=(1,1)^{T}$, $\mathbf{a}_{1}^{-}=(1,-1)^{T}$, and the eigenvalues corresponding to them have the form $\mu_{1}^{+}=a_{0}+a_{1}$, $\mu_{1}^{-}=a_{0}-a_{1}$. For the matrix:

$$
A_{2}=\left(\begin{array}{llll}
a_{0} & a_{1} & a_{2} & a_{3} \\
a_{1} & a_{0} & a_{3} & a_{2} \\
a_{2} & a_{3} & a_{0} & a_{1} \\
a_{3} & a_{2} & a_{1} & a_{0}
\end{array}\right)=\left(\begin{array}{ll}
A_{1}\left(a_{0}, a_{1}\right) & A_{1}\left(a_{2}, a_{3}\right) \\
A_{1}\left(a_{2}, a_{3}\right) & A_{1}\left(a_{0}, a_{1}\right)
\end{array}\right)
$$

the eigenvectors have the form:

$$
\mathbf{a}_{2}^{(+,+)}=\left(\mathbf{a}_{1}^{+}, \mathbf{a}_{1}^{+}\right)^{T}, \mathbf{a}_{2}^{(-,+)}=\left(\mathbf{a}_{1}^{-}, \mathbf{a}_{1}^{-}\right)^{T}, \mathbf{a}_{2}^{(+,-)}=\left(\mathbf{a}_{1}^{+},-\mathbf{a}_{1}^{+}\right)^{T}, \mathbf{a}_{2}^{(-,-)_{2}}=\left(\mathbf{a}_{1}^{-},-\mathbf{a}_{1}^{-}\right)^{T},
$$

or briefly, $\mathbf{a}_{2}^{\left( \pm_{1}, \pm_{2}\right)}=\left(\mathbf{a}_{1}^{ \pm_{1}}, \pm_{2} \mathbf{a}_{1}^{ \pm_{1}}\right)^{T}$. The + and - signs in the expressions $\pm_{1}$ and $\pm_{2}$ are values taken independently of each other. Indeed, the equalities:

$$
\begin{gathered}
A_{2} \mathbf{a}_{2}^{\left( \pm_{1}, \pm_{2}\right)}=\left(\begin{array}{ll}
A_{1}\left(a_{0}, a_{1}\right) & A_{1}\left(a_{2}, a_{3}\right) \\
A_{1}\left(a_{2}, a_{3}\right) & A_{1}\left(a_{0}, a_{1}\right)
\end{array}\right)\left(\begin{array}{c}
\mathbf{a}_{1}^{ \pm_{1}} \\
\pm_{2} \mathbf{a}_{1}^{ \pm_{1}}
\end{array}\right)=\left(\begin{array}{c}
A_{1}\left(a_{0}, a_{1}\right) \mathbf{a}_{1}^{ \pm_{1}} \pm_{2} A_{1}\left(a_{2}, a_{3}\right) \mathbf{a}_{1}^{ \pm_{1}} \\
A_{1}\left(a_{2}, a_{3}\right) \mathbf{a}_{1}^{ \pm_{1}} \pm_{2} A_{1}\left(a_{0}, a_{1}\right) \mathbf{a}_{1}^{ \pm_{1}}
\end{array}\right) \\
=\left(\begin{array}{c}
\left(a_{0} \pm_{1} a_{1}\right) \mathbf{a}_{1}^{ \pm_{1}} \pm_{2}\left(a_{2} \pm_{1} a_{3}\right) \mathbf{a}_{1}^{ \pm_{1}} \\
\left(a_{2} \pm_{1} a_{3}\right) \mathbf{a}_{1}^{ \pm_{1}} \pm_{2}\left(a_{0} \pm_{1} a_{1}\right) \mathbf{a}_{1}^{ \pm_{1}}
\end{array}\right)=\left(a_{0} \pm_{1} a_{1} \pm_{2}\left(a_{2} \pm_{1} a_{3}\right)\right)\left(\begin{array}{c}
\mathbf{a}_{1}^{ \pm_{1}} \\
\pm_{2} \mathbf{a}_{1}^{ \pm_{1}}
\end{array}\right) \\
=\left(a_{0} \pm_{1} a_{1} \pm_{2}\left(a_{2} \pm_{1} a_{3}\right)\right) \mathbf{a}_{2}^{\left( \pm_{1}, \pm_{2}\right)}
\end{gathered}
$$

are true, and hence, $\left(\mathbf{a}_{1}^{ \pm_{1}}, \pm_{2} \mathbf{a}_{1}^{ \pm_{1}}\right)^{T}$ are the eigenvectors for four different combinations of signs $\pm_{1}$ and $\pm_{2}$. It is seen that the eigenvectors $\mathbf{a}_{2}^{\left( \pm_{1}, \pm_{2}\right)}=\left(1, \pm_{1} 1, \pm_{2} 1, \pm_{2} \pm_{1} 1\right)^{T}$ of the matrix $A_{2}\left(a_{0}, a_{1}, a_{2}, a_{3}\right)$ do not depend on numbers $\left\{a_{k}\right\}$. 
Further, assuming that the eigenvectors $\mathbf{a}_{n-1}^{0}, \ldots, \mathbf{a}_{n-1}^{2^{n-1}-1}$ of the matrix $A_{n-1}\left(a_{0}, \ldots, a_{2^{n-1}-1}\right)$ do not depend on its coefficients, we prove that this property is also true for the matrix $A_{n}\left(a_{0}, \ldots, a_{2^{n}-1}\right)$.

Let $\mu_{n-1}^{0}, \ldots, \mu_{n-1}^{2^{n-1}-1}$ be the eigenvalues corresponding to the above eigenvectors of the matrix $A_{n-1}\left(a_{0}, \ldots, a_{2^{n-1}-1}\right)$, independent of its coefficients, then vectors of the form $\mathbf{a}_{n}^{k}=\left(\mathbf{a}_{n-1}^{k}, \pm \mathbf{a}_{n-1}^{k}\right)^{T}$, where $k=0, \ldots, 2^{n-1}-1$, are the eigenvectors of the matrix $A_{n}\left(a_{0}, \ldots, a_{2^{n}-1}\right)$. Indeed, we have:

$$
\begin{gathered}
A_{n} \mathbf{a}_{n}^{k}=A_{n}\left(\mathbf{a}_{n-1}^{k} \pm \mathbf{a}_{n-1}^{k}\right)=\left(\begin{array}{cc}
A_{n-1}\left(a_{0}, \ldots, a_{2^{n-1}-1}\right) & A_{n-1}\left(a_{2^{n-1}}, \ldots, a_{2^{n}-1}\right) \\
A_{n-1}\left(a_{2^{n-1}}, \ldots, a_{2^{n}-1}\right) & A_{n-1}\left(a_{0}, \ldots, a_{2^{n-1}-1}\right)
\end{array}\right)\left(\begin{array}{c}
\mathbf{a}_{n-1}^{k} \\
\pm \mathbf{a}_{n-1}^{k}
\end{array}\right) \\
=\left(\begin{array}{c}
A_{n-1}\left(a_{0}, \ldots, a_{2^{n-1}-1}\right) \mathbf{a}_{n-1}^{k} \pm A_{n-1}\left(a_{2^{n-1}}, \ldots, a_{2^{n}-1}\right) \mathbf{a}_{n-1}^{k} \\
A_{n-1}\left(a_{2^{n-1}}, \ldots, a_{2^{n}-1}\right) \mathbf{a}_{n-1}^{k} \pm A_{n-1}\left(a_{0}, \ldots, a_{2^{n-1}-1}\right) \mathbf{a}_{n-1}^{k}
\end{array}\right) \\
=\left(\begin{array}{c}
\mu_{n-1}^{k} \mathbf{a}_{n-1}^{k} \pm \hat{\mu}_{n-1}^{k} \mathbf{a}_{n-1}^{k} \\
\hat{\mu}_{n-1}^{k} \mathbf{a}_{n-1}^{k} \pm \mu_{n-1}^{k} \mathbf{a}_{n-1}^{k}
\end{array}\right)=\left(\begin{array}{l}
\mu_{n-1}^{k} \pm \hat{\mu}_{n-1}^{k}
\end{array}\right)\left(\begin{array}{c}
\mathbf{a}_{n-1}^{k} \\
\pm \mathbf{a}_{n-1}^{k}
\end{array}\right)=\left(\begin{array}{l}
\mu_{n-1}^{k} \pm \hat{\mu}_{n-1}^{k}
\end{array}\right) \mathbf{a}_{n}^{k},
\end{gathered}
$$

where $\hat{\mu}_{n-1}^{k}$ is the eigenvalue of the matrix $A_{n-1}\left(a_{2^{n-1}}, \ldots, a_{2^{n}-1}\right)$ corresponding to the eigenvector $\mathbf{a}_{n-1}^{k}$. Obviously, there are $2^{n}$ vectors of the form $\mathbf{a}_{n}^{k}=\left(\mathbf{a}_{n-1}^{k}, \pm \mathbf{a}_{n-1}^{k}\right)^{T}$. Therefore, the eigenvalues of the matrix $A_{n}\left(a_{0}, \ldots, a_{2^{n}-1}\right)$ are $\mu_{n}^{k, \pm}=\mu_{n-1}^{k} \pm \hat{\mu}_{n-1}^{k}$.

Orthogonality: It is obvious that the eigenvectors $\mathbf{a}_{1}^{+}=(1,1)^{T}, \mathbf{a}_{1}^{-}=(1,-1)^{T}$ of the matrix $A_{1}\left(a_{0}, a_{1}\right)$ are orthogonal. If the eigenvectors $\mathbf{a}_{n-1}^{k}, k=0, \ldots, 2^{n-1}-1$ of the matrix $A_{n-1}\left(a_{2^{n-1}}, \ldots, a_{2^{n}-1}\right)$ are chosen as orthogonal, then the eigenvectors $\mathbf{a}_{n}^{k}=\left(\mathbf{a}_{n-1}^{k}, \pm \mathbf{a}_{n-1}^{k}\right)^{T}$ of the matrix $A_{n}\left(a_{0}, \ldots, a_{2^{n}-1}\right)$ are also orthogonal:

$$
\mathbf{a}_{n}^{k_{1}} \mathbf{a}_{n}^{k_{2}}=\left(\mathbf{a}_{n-1}^{k_{1}}, \pm \mathbf{a}_{n-1}^{k_{1}}\right)^{T}\left(\mathbf{a}_{n-1}^{k_{2}}, \pm \mathbf{a}_{n-1}^{k_{2}}\right)^{T}=\mathbf{a}_{n-1}^{k_{1}} \mathbf{a}_{n-1}^{k_{2}}+\mathbf{a}_{n-1}^{k_{1}} \mathbf{a}_{n-1}^{k_{2}}=0, k_{1} \neq k_{2}
$$

and $\left(\mathbf{a}_{n-1}^{k}, \mathbf{a}_{n-1}^{k}\right)^{T}\left(\mathbf{a}_{n-1}^{k},-\mathbf{a}_{n-1}^{k}\right)^{T}=0$. The theorem is proven.

Corollary 3. Let $k=\left(k_{n}, \ldots, k_{1}\right)_{2}, k_{i}=0,1$, then the eigenvector of the matrix $A_{n}$ numbered by $k$ can be written in the form:

$$
\begin{aligned}
& \mathbf{a}_{n}^{k}=\left(1,(-1)^{k_{1}},(-1)^{k_{2}},(-1)^{k_{2}+k_{1}},(-1)^{k_{3}},(-1)^{k_{3}+k_{1}},(-1)^{k_{3}+k_{2}},\right. \\
& \left.(-1)^{k_{3}+k_{2}+k_{1}},(-1)^{k_{4}}, \ldots,(-1)^{k_{n}+\ldots+k_{1}}\right)^{T}=\left((-1)^{k \otimes m}\right)_{m=0, \ldots, 2^{n}-1^{\prime}}
\end{aligned}
$$

where $k \otimes i \equiv\left(k_{n} \ldots k_{1}\right)_{2} \otimes\left(i_{n} \ldots i_{1}\right)_{2}=k_{n} i_{n}+\ldots+k_{1} i_{1}$ is a "scalar" product of the indexes $(k)_{2}$ and $(i)_{2}$.

The eigenvalue corresponding to the eigenvector $\mathbf{a}_{n}^{\left(k_{n} \ldots k_{1}\right)_{2}}$ can be written in a similar form:

$$
\mu_{n}^{k} \equiv \mu_{n}^{\left(k_{n} \ldots k_{1}\right)_{2}}=\sum_{i=0}^{2^{n}-1}(-1)^{k \otimes i} a_{i}=\sum_{i=0}^{2^{n}-1}(-1)^{k_{n} i_{n}+\ldots+k_{1} i_{1}} a_{\left(i_{n} \ldots i_{1}\right)_{2}} .
$$


Proof. Let us prove (13). For $n=1$, we have $\mathbf{a}_{1}^{+}=\mathbf{a}_{1}^{(0)_{2}}=\left((-1)^{0 \otimes 0},(-1)^{0 \otimes 1}\right)^{T}$, $\mathbf{a}_{1}^{-}=\mathbf{a}_{1}^{(1)_{2}}=\left((-1)^{1 \otimes 0},(-1)^{1 \otimes 1}\right)^{T}$, and (13) is true. If Formula (13) is true for the vector $\mathbf{a}_{n-1}^{\left(k_{n-1} \ldots k_{1}\right)_{2}}$, then by Theorem 3 , we have:

$$
\begin{gathered}
\left(\mathbf{a}_{n-1}^{\left(k_{n-1} \ldots k_{1}\right)_{2}}, \pm \mathbf{a}_{n-1}^{\left(k_{n-1} \ldots k_{1}\right)_{2}}\right)^{T}=\left(\mathbf{a}_{n-1}^{\left(k_{n-1} \ldots k_{1}\right)_{2}},(-1)^{k_{n}} \mathbf{a}_{n-1}^{\left(k_{n-1} \ldots k_{1}\right)_{2}}\right)^{T} \\
=\left(\left((-1)^{\left(k_{n} k_{n-1} \ldots k_{1}\right)_{2} \otimes\left(0 m_{n-1} \ldots m_{1}\right)_{2}}\right)_{m=0, \ldots, 2^{n-1}-1^{\prime}}\right. \\
\left.\left((-1)^{\left(k_{n} k_{n-1} \ldots k_{1}\right)_{2} \otimes\left(1 m_{n-1} \ldots m_{1}\right)_{2}}\right)_{m=0, \ldots, 2^{n-1}-1}\right)^{T} \\
=\left(\left((-1)^{k \otimes m}\right)_{m=0, \ldots, 2^{n-1}-1^{\prime}}\left((-1)^{k \otimes m}\right)_{m=2^{n-1}, \ldots, 2^{n}-1}\right)^{T} \\
=\left(\left((-1)^{k \otimes m}\right)_{m=0, \ldots, 2^{n}-1}\right)^{T}=\mathbf{a}_{n}^{\left(k_{n} k_{n-1} \ldots k_{1}\right)_{2}}
\end{gathered}
$$

and hence, the formula (13) is also true for the vector $\mathbf{a}_{n}^{\left(k_{n} k_{n-1} \ldots k_{1}\right)_{2}}=\mathbf{a}_{n}^{k}$.

Let us prove (14). For $n=1$, we have:

$$
\mu_{1}^{k_{1}}=a_{0}+(-1)^{k_{1}} a_{1}=(-1)^{0} a_{(0)_{2}}+(-1)^{k_{1} \cdot 1} a_{(1)_{2}},
$$

where $k_{1}=0,1$. Assume that the formula (14) is valid for $n=n-1$, and prove its validity for $n$. By Theorem 3, changing the notation $\pm=(-1)^{k_{n}}$, we write:

$$
\begin{gathered}
\mu_{n}^{k, \pm}=\mu_{n-1}^{k} \pm \hat{\mu}_{n-1}^{k}=\sum_{i=0}^{2^{n-1}-1}(-1)^{k_{n-1} i_{n-1}+\ldots+k_{1} i_{1}} a_{\left(i_{n-1} \ldots i_{1}\right)_{2}} \\
+\sum_{i=0}^{2^{n-1}-1}(-1)^{k_{n} \cdot 1+k_{n-1} i_{n-1}+\ldots+k_{1} i_{1}} a_{\left(1 i_{n-1} \ldots i_{1}\right)_{2}}=\sum_{i=0}^{2^{n-1}-1}(-1)^{k_{n} \cdot i_{n}+k_{n-1} i_{n-1}+\ldots+k_{1} i_{1}} a_{\left(i_{n} i_{n-1} \ldots i_{1}\right)_{2}} \\
+\sum_{i=2^{n-1}}^{2^{n}-1}(-1)^{k_{n} \cdot i_{n}+k_{n-1} i_{n-1}+\ldots+k_{1} i_{1}} a_{\left(i_{n} i_{n-1} \ldots i_{1}\right)_{2}}=\sum_{i=0}^{2^{n}-1}(-1)^{k_{n} \cdot i_{n}+\ldots+k_{1} i_{1}} a_{\left(i_{n} i_{n-1} \ldots i_{1}\right)_{2}}
\end{gathered}
$$

which proves (14). The corollary is proven.

Let us denote the operation of taking the adjoint matrix to the matrix $A_{n}\left(a_{0}, \ldots, a_{2^{n}-1}\right)$, for the convenience of presentation, in the form $\operatorname{adj}\left(A_{n}\left(a_{0}, \ldots, a_{2^{n}-1}\right)\right)=\overline{A_{n}}\left(a_{0}, \ldots, a_{2^{n}-1}\right)$. Then, by the definition of the adjoint matrix:

$$
A_{n}\left(a_{0}, \ldots, a_{2^{n}-1}\right) \overline{A_{n}}\left(a_{0}, \ldots, a_{2^{n}-1}\right)=\left(\operatorname{det} A_{n}\right) I_{2^{n}} .
$$

In the case $n=0$, we assume that $\overline{a_{k}}=1$. Obviously, $\overline{\overline{A_{n}}}=A_{n}$, and if the matrices $A_{n}$ and $B_{n}$ of the form of (6) are nonsingular, then, by virtue of Theorem 2, on the commutativity of $A_{n}$ and $B_{n}$, we have:

$$
\begin{gathered}
\overline{A_{n} B_{n}}=\overline{B_{n} A_{n}}=\operatorname{det}\left(B_{n} A_{n}\right)\left(B_{n} A_{n}\right)^{-1}=\operatorname{det}\left(B_{n} A_{n}\right) A_{n}{ }^{-1} B_{n}{ }^{-1} \\
=\operatorname{det}\left(A_{n}\right) A_{n}{ }^{-1} \operatorname{det}\left(B_{n}\right) B_{n}{ }^{-1}=\overline{A_{n} B_{n}}
\end{gathered}
$$

Define the operation of multiplying a matrix $C_{k-1}$ by a block matrix $\left(\begin{array}{cc}A_{k-1}^{0} & A_{k-1}^{1} \\ A_{k-1}^{1} & A_{k-1}^{0}\end{array}\right)$ in the form:

$$
C_{k-1} \cdot\left(\begin{array}{cc}
A_{k-1}^{0} & A_{k-1}^{1} \\
A_{k-1}^{1} & A_{k-1}^{0}
\end{array}\right) \equiv\left(\begin{array}{cc}
C_{k-1} & 0 \\
0 & C_{k-1}
\end{array}\right)\left(\begin{array}{cc}
A_{k-1}^{0} & A_{k-1}^{1} \\
A_{k-1}^{1} & A_{k-1}^{0}
\end{array}\right)=\left(\begin{array}{ll}
C_{k-1} A_{k-1}^{0} & C_{k-1} A_{k-1}^{1} \\
C_{k-1} A_{k-1}^{1} & C_{k-1} A_{k-1}^{0}
\end{array}\right)
$$


Note that if $C_{k-1}=C_{k-1}\left(c_{0}, \ldots, c_{2^{k-1}-1}\right)$, then $C_{k}=C_{k}\left(c_{0}, \ldots, c_{2^{k-1}-1}, 0, \ldots, 0\right)$ :

$$
C_{k-1} \cdot\left(\begin{array}{cc}
A_{k-1}^{0} & A_{k-1}^{1} \\
A_{k-1}^{1} & A_{k-1}^{0}
\end{array}\right)=\left(\begin{array}{cc}
C_{k-1} & 0 \\
0 & C_{k-1}
\end{array}\right)\left(\begin{array}{cc}
A_{k-1}^{0} & A_{k-1}^{1} \\
A_{k-1}^{1} & A_{k-1}^{0}
\end{array}\right)=C_{k} A_{k}=A_{k} C_{k}=A_{k} \cdot C_{k-1}
$$

and hence, by Theorem 2, the matrix $C_{k-1} \cdot A_{k}$ has the form of (6).

Theorem 4. The determinant of the matrix $A_{n}\left(a_{0}, \ldots, a_{2^{n}-1}\right)$ can be written in the form:

$$
\operatorname{det} A_{n}\left(a_{0}, \ldots, a_{2^{n}-1}\right)=\prod_{k=0}^{2^{n}-1}\left(\sum_{i=0}^{2^{n}-1}(-1)^{k_{n} i_{n}+\ldots+k_{1} i_{1}} a_{\left(i_{n} \ldots i_{1}\right)_{2}}\right) \text {. }
$$

For any $m=0, \ldots, n$, the equality holds:

$$
\begin{aligned}
\overline{A_{n}}\left(a_{0}, \ldots, a_{2^{n}-1}\right)=\overline{\left(\operatorname{det} A_{n-m}\right)} & \left(A_{m}^{(0 \ldots 0)_{2}}, \ldots, A_{m}^{\left(k_{n} \ldots k_{m+1}\right)_{2}}, \ldots, A_{m}^{(1 \ldots 1)_{2}}\right) \\
& \times \overline{A_{n-m}}\left(A_{m}^{(0 \ldots)_{2}}, \ldots, A_{m}^{\left(k_{n} \ldots k_{m+1}\right)_{2}}, \ldots, A_{m}^{(1 \ldots 1)_{2}}\right),
\end{aligned}
$$

where $\operatorname{det} A_{n-m}$ is calculated for the block matrix $A_{n-m}$, first with numerical coefficients a $\left.a_{n} \ldots k_{m+1}\right)_{2}$, then the corresponding matrices $A_{m}^{\left(k_{n} \ldots k_{m+1}\right)_{2}}$ are substituted for them, and the adjoint matrix is taken for the resulting matrix. In addition, the adjoint matrix $\overline{A_{n-m}}$ is also constructed for numerical coefficients $a^{\left(k_{n} \ldots k_{m+1}\right)_{2}}$, and then, the corresponding matrices $A_{m}^{\left(k_{n} \ldots k_{m+1}\right)_{2}}$ are substituted for them.

Proof. Since the determinant of a matrix is equal to the product of its eigenvalues, using Formula (14), we obtain:

$$
\operatorname{det} A_{n}\left(a_{0}, \ldots, a_{2^{n}-1}\right)=\prod_{k=0}^{2^{n}-1} \mu_{n}^{\left(k_{n} \ldots k_{1}\right)_{2}}=\prod_{k=0}^{2^{n}-1}\left(\sum_{i=0}^{2^{n}-1}(-1)^{k_{n} i_{n}+\ldots+k_{1} i_{1}} a_{\left(i_{n} \ldots i_{1}\right)_{2}}\right) .
$$

Let us prove (17). Note that all operations for constructing matrices in this formula are correct since the determinant of a matrix and the elements of an adjoint matrix are algebraic expressions and all matrices involved in these constructions are commutative.

Denote the matrix on the right side of (17) by $M$, and multiply it on the right by the matrix $A_{n}$ represented in the form of (10), then substitute numbers $a^{\left(k_{n} \ldots k_{m+1}\right)_{2}}$ instead of matrices $A_{m}^{\left(k_{n} \ldots k_{m+1}\right)_{2}}$; after transformations, return the matrices $A_{m}^{\left(k_{n} \ldots k_{m+1}\right)_{2}}$ back:

$$
\begin{gathered}
M A_{n}\left(a_{0}, \ldots, a_{2^{n}-1}\right)=M A_{n-m}\left(A_{m}^{(0 \ldots 0)_{2}}, \ldots, A_{m}^{(1 \ldots 1)_{2}}\right) \\
=\overline{\left(\operatorname{det} A_{n-m}^{b}\right)\left(a^{(0 \ldots 0)_{2}}, \ldots, a^{\left(k_{n} \ldots k_{m+1}\right)_{2}}, \ldots, a^{(1 \ldots 1)_{2}}\right)} \\
\overline{A^{b}{ }_{n-m}}\left(a^{(0 \ldots 0)_{2}}, \ldots, a^{\left.\left(k_{n} \ldots k_{m+1}\right)_{2}, \ldots, a^{(1 \ldots 1)_{2}}\right)}\right. \\
A_{n-m}^{b}\left(a^{\left.(0 \ldots 0)_{2}, \ldots, a^{\left(k_{n} \ldots k_{m+1}\right)_{2}}, \ldots, a^{(1 \ldots 1)_{2}}\right)}=\left(\overline{\operatorname{det} A_{n-m}} \cdot I_{2^{n-m}}^{b}\right)\left(\operatorname{det} A_{n-m} \cdot I_{2^{n-m}}^{b}\right)\right. \\
=\prod_{k=0}^{2^{n-m}-1} \overline{\left(\sum_{i=0}^{2^{n-m}-1}(-1)^{k \otimes i} A_{m}^{\left(i_{n-m} \ldots i_{1}\right)_{2}}\right)}\left(\sum_{i=0}^{2^{n-m}-1}(-1)^{k \otimes i} A_{m}^{\left(i_{n-m} \ldots i_{1}\right)_{2}}\right) \cdot I_{2^{n-m}}^{b} \\
=\prod_{k=0}^{2^{n-m}-1} \operatorname{det}\left(\sum_{i=0}^{2^{n-m}-1}(-1)^{k_{n-m} i_{n-m}+\ldots+k_{1} i_{1}} A_{m}^{\left(i_{n-m} \ldots i_{1}\right)_{2}}\right) I_{2^{m}} \cdot I_{2^{n-m}}^{b} \\
=\prod_{k=0}^{2^{n-m}-1} \operatorname{det}\left(\sum_{i=0}^{2^{n-m}-1}(-1)^{k_{n-m} i_{n-m}+\ldots+k_{1} i_{1}} A_{m}^{\left(i_{n-m} \ldots i_{1}\right)_{2}}\right) I_{2^{n}} \equiv \lambda I_{2^{n}},
\end{gathered}
$$


where the formula (16) and an equality of the form of (15) are used and $\lambda \in \mathbb{R}$. Here, the matrix $I_{n-m}^{b}$ has a superscript $b$ indicating that it is a block matrix, each block of size $2^{m} \times 2^{m}$. The resulting equality proves (17). The theorem is proven.

Corollary 4. The matrix $\overline{A_{n}}\left(a_{0}, \ldots, a_{2^{n}-1}\right)$ is a matrix of the form of (6). If inverse matrix $A_{n}^{-1}\left(a_{0}, \ldots, a_{2^{n}-1}\right)$ exists, then it also has the form of (6).

Proof. Obviously, $\overline{A_{1}}\left(a_{0}, a_{1}\right)=A_{1}\left(a_{0},-a_{1}\right)$, and therefore, the matrix $\overline{A_{1}}\left(a_{0}, a_{1}\right)$ is of the form of (6). Suppose the matrix $\overline{A_{n-1}}\left(a_{0}, \ldots, a_{2^{n-1}-1}\right)$ for $n>1$ is of the form of (6). Then, using Formula (17) for $m=n-1$, we obtain:

$$
\begin{gathered}
\overline{A_{n}}=\overline{\left(\operatorname{det} A_{1}\right)}\left(A_{n-1}^{0}, A_{n-1}^{1}\right) \cdot \overline{A_{1}}\left(A_{n-1}^{0}, A_{n-1}^{1}\right) \\
=\overline{\left(\left(A_{n-1}^{0}\right)^{2}-\left(A_{n-1}^{1}\right)^{2}\right)} \cdot A_{1}\left(A_{n-1}^{0},-A_{n-1}^{1}\right) .
\end{gathered}
$$

It is clear that the matrix $\operatorname{det} A_{1}\left(A_{n-1}^{0}, A_{n-1}^{1}\right)=\left(A_{n-1}^{0}\right)^{2}-\left(A_{n-1}^{1}\right)^{2}$ has the form of (6), and hence, by the induction hypothesis, the matrix $\overline{\left(\left(A_{n-1}^{0}\right)^{2}-\left(A_{n-1}^{1}\right)^{2}\right)}$ is also a matrix of the form of (6). Since, as said before in Theorem 4, the matrix $C_{n-1} \cdot A_{n}$ has the form of (6), and the product of the matrices of the form of (6) is again a matrix of the form of (6). The induction step is proven, and therefore, the corollary is true.

Corollary 5. The matrix $\overline{A_{n}}\left(a_{0}, \ldots, a_{2^{n}-1}\right)$ can be calculated using the recurrent formula:

$$
\begin{aligned}
& \overline{A_{n}}\left(a_{0}, \ldots, a_{2^{n}-1}\right)= \overline{\left(\operatorname{det} A_{n-1}\right)}\left(A_{1}^{(0 \ldots 0)_{2}}, \ldots, A_{1}^{\left(k_{n} \ldots k_{2}\right)_{2}}, \ldots, A_{1}^{(1 \ldots 1)_{2}}\right) \\
& \times \overline{A_{n-1}}\left(A_{1}^{(0 . \ldots 0)_{2}}, \ldots, A_{1}^{\left(k_{n} \ldots k_{2}\right)_{2}}, \ldots, A_{1}^{(1 \ldots 1)_{2}}\right) \\
&=\left.\prod_{\left(k_{n} \ldots k_{2}\right)_{2}=0}^{2^{n-1}-1}\left(\sum_{\left(i_{n} \ldots i_{2}\right)_{2}=0}^{2^{n-1}-1}(-1)^{k_{n} i_{n}+\ldots+k_{2} i_{2}} \overline{A_{1}^{\left(i_{n} \ldots i_{2}\right)_{2}}}\right) \overline{A_{n-1}}\left(b_{0}, \ldots, b_{2^{n-1}-1}\right)\right|_{b_{k}=A_{1}^{k^{\prime}}}
\end{aligned}
$$

where $A_{1}^{k}=A_{1}^{\left(k_{n} \ldots k_{2}\right)_{2}}=A_{1}\left(a_{\left(k_{n} \ldots k_{2} 0\right)_{2}}, a_{\left(k_{n} \ldots k_{2} 1\right)_{2}}\right)$.

This equality follows from (17) when $m=n-1$, taking into account (16) and the fact that $\overline{A_{1}+B_{1}}=\overline{A_{1}}+\overline{B_{1}}$.

\section{Properties of Integrodifferential Operators}

In this section, we present some well-known statements about fractional integrodifferential operators introduced in Section 1. In [27], the following assertions were proven.

Lemma 1. Let $\alpha>0, \mu \geq 0,0<\lambda<1$ and $u(x) \in C^{\lambda+p}(\bar{\Omega}), p \in N_{0}$. Then:

(1) If $\mu>0$, then $J_{\mu}^{\alpha}[u](x) \in C^{\lambda+p}(\bar{\Omega})$;

(2) If $\mu=0$, then if the condition $u(0)=0$ holds, the function $J_{0}^{\alpha}[u](x)$ also belongs to the class $C^{\lambda+p}(\bar{\Omega})$, and the equality $J_{0}^{\alpha}[u](0)=0$ is valid.

Lemma 2. Let $\mu \geq 0, p-1<\alpha \leq p, p=1,2, \ldots, 0<\lambda<1$, and $u(x) \in C^{\lambda+q}(\bar{\Omega}), q \geq p$. Then, the function $D_{\mu}^{\alpha}[u](x)$ belongs to the class $C^{\lambda+q-p}(\bar{\Omega})$, and the equality $D_{0}^{\alpha}[u](0)=0$ holds.

Lemma 3. Let $\mu \geq 0, p-1<\alpha \leq p, p=1,2, \ldots, 0<\lambda<1$ and $u(x) \in C^{\lambda+q}(\bar{\Omega}), q \geq l$. Then, for any $x \in \bar{\Omega}$, the following equality holds:

$$
J_{\mu}^{\alpha}\left[D_{\mu}^{\alpha}[u]\right](x)=\left\{\begin{array}{l}
u(x), \mu>0 \\
u(x)-u(0), \mu=0
\end{array}\right.
$$


Lemma 4. Let $\mu \geq 0, p-1<\alpha \leq p, p=1,2, \ldots, 0<\lambda<1$ and $u(x) \in C^{\lambda+q}(\bar{\Omega}), q \geq p$. Then, for any $x \in \bar{\Omega}$ for $\mu>0$, the following equality holds:

$$
D_{\mu}^{\alpha}\left[J_{\mu}^{\alpha}[u]\right](x)=u(x)
$$

For $\mu=0$, the equality (19) is valid under the additional condition $u(0)=0$.

Lemma 5. Let $\mu \geq 0, p-1<\alpha \leq p, p=1,2, \ldots, f(x)$ be a smooth function in the domain $\bar{\Omega}$ and $-\Delta u(x)=f(x), x \in \Omega$. Then, the equality is valid:

$$
-\Delta D_{\mu}^{\alpha}[u](x)=F(x), x \in \Omega,
$$

where:

$$
F(x)=D_{\mu+2}^{\alpha}[f](x) .
$$

Lemma 6. If $\mu=0,0<\alpha \leq 1$, then function $F(x)$ from the equality (21) can be represented as:

$$
F(x)=\left(r \frac{d}{d r}+4\right) f_{1-\alpha}(x),
$$

where:

$$
f_{1-\alpha}(x)=J_{4}^{1-\alpha}[f](x)
$$

\section{The Dirichlet Problem}

In this section, we study the problem $H$ for $\alpha=0$, i.e., the following Dirichlet problem:

$$
\begin{gathered}
L_{n} u(x)=f(x), x \in \Omega, \\
u(x)=g_{0}(x), \quad \frac{\partial u(x)}{\partial v}=g_{1}(x), x \in \partial \Omega .
\end{gathered}
$$

In [20], the following assertion was proven.

Lemma 7. ([20], Lemma 3.1) Let $S$ be an orthogonal matrix, then the operator $I_{S} u(x)=u(S x)$ and the Laplace operator $\Delta$ commute $\Delta I_{S} u(x)=I_{S} \Delta u(x)$ on functions $u \in C^{2}(\Omega)$. The operator $\delta u(x)=\sum_{i=1}^{n} x_{i} u_{x_{i}}(x)$ and the operator $I_{S}$ also commute $\delta I_{S} u(x)=I_{S} \delta u(x)$ on functions $u \in C^{1}(\bar{\Omega})$, and the equality $\nabla I_{S}=I_{S} S^{T} \nabla$ is valid.

Corollary 6. If the function $u(x)$ is biharmonic in the domain $\Omega$, then the function $u\left(S_{n}^{i_{n}} \ldots S_{1}^{i_{1}} x\right)=$ $I_{S_{n}^{i_{n}} \ldots S_{1}^{i_{1}}} u(x)$, and hence, the function $v(x)$ from (4) is also biharmonic in $\Omega$.

Proof. Indeed, the matrix $S_{n}^{i_{n}} \ldots S_{1}^{i_{1}}$ is symmetric and orthogonal as $\left(S_{n}^{i_{n}} \ldots S_{1}^{i_{1}}\right)^{2}=I$, and hence, by virtue of Lemma 7:

$$
\Delta^{2} u(x)=0 \Rightarrow \Delta^{2} I_{S_{n}^{i_{n}} \ldots S_{1}^{i_{1}}} u(x)=I_{S_{n}^{i_{n}} \ldots S_{1}^{i_{1}}} \Delta^{2} u(x)=0
$$

and $\Delta^{2} v(x)=0$. It follows that if the function $u(x)$ is biharmonic in $\Omega$, it satisfies the homogeneous Equation (1) in $\Omega$. The corollary is proven.

The converse assertion is also valid.

Lemma 8. Let the function $u \in C^{4}(\Omega)$ satisfy homogeneous Equation (1) and $\sum_{i=0}^{2^{n}-1}(-1)^{k \otimes i} a_{i} \neq 0$, for $k=0, \ldots, 2^{n}-1$, then the function $u(x)$ is biharmonic in $\Omega$. 
Proof. Let the function $u \in C^{4}(\Omega)$ satisfy homogeneous Equation (1). Consider the function $v(x)$ from (4):

$$
v(x)=\sum_{i \equiv\left(i_{n} \ldots i_{1}\right)_{2}=0}^{(1 \ldots 1)_{2}} a_{i} u\left(S_{n}^{i_{n}} \ldots S_{1}^{i_{1}} x\right) .
$$

It is evident that $v(x) \in C^{4}(\Omega)$ and $\Delta^{2} v(x)=0, x \in \Omega$, i.e., the function $v(x)$ is biharmonic in $\Omega$. By virtue of Corollary 6, functions $v\left(S_{n}^{i_{n}} \ldots S_{1}^{i_{1}} x\right)$ are also biharmonic in $\Omega$. Further, as stated in (5), for vectors:

$$
\begin{aligned}
& U(x)=\left(u(x), \ldots, u\left(S_{n}^{i_{n}} \ldots S_{1}^{i_{1}} x\right), \ldots, u\left(S_{n}^{1} \ldots S_{1}^{1} x\right)\right)^{T}, \\
& V(x)=\left(v(x), \ldots, v\left(S_{n}^{j_{n}} \ldots S_{1}^{j_{1}} x\right), \ldots, v\left(S_{n}^{1} \ldots S_{1}^{1} x\right)\right)^{T}
\end{aligned}
$$

the equality $V(x)=A_{n} U(x)$ holds, where $A_{n}=A_{n}\left(a_{0}, \ldots, a_{2^{n}-1}\right)$ is a matrix of the form of (6). By the condition of the lemma and by virtue of Theorem 4, the determinant of this system does not vanish. By Corollary 4 , the matrix $A_{n}{ }^{-1}$ also has the form of (6). Let us introduce the notation $B_{n}\left(b_{0}, \ldots, b_{2^{n}-1}\right)=A_{n}{ }^{-1}\left(a_{0}, \ldots, a_{2^{n}-1}\right)$. If the first row of the matrix $B_{n}$ is written as $b=\left(b_{0}, \ldots, b_{2^{n}-1}\right)^{T}$, we obtain:

$$
u(x)=b \cdot V(x)=\sum_{i \equiv\left(i_{n} \ldots i_{1}\right)_{2}=0}^{(1 \ldots 1)_{2}} b_{i} v\left(S_{n}^{i_{n}} \ldots S_{1}^{i_{1}} x\right) .
$$

This immediately implies that the function $u(x)$ is biharmonic in $\Omega$. The lemma is proven.

Theorem 5. If the coefficients of the operator $L_{n}$ satisfy the condition $\sum_{i=0}^{2^{n}-1}(-1)^{k \otimes i} a_{i} \neq 0$, for $k=0, \ldots, 2^{n}-1$ and a solution to the Dirichlet problem (24) and (25) exists, then it is unique.

Proof. Let us prove that the homogeneous problem (24) and (25) has only a zero solution, and hence, the solution to the inhomogeneous problem (24) and (25), if it exists, is unique. Let $u(x)$ be a solution to the homogeneous problem (24) and (25). By Lemma 8 , the function $u(x)$ is biharmonic in $\Omega$ and satisfies the homogeneous conditions (24). Therefore, the function $u(x)$ is a solution to the following Dirichlet problem:

$$
\Delta^{2} u(x)=0, x \in \Omega ;\left.\quad u(x)\right|_{\partial \Omega}=0,\left.\frac{\partial u(x)}{\partial v}\right|_{\partial \Omega}=0 .
$$

By virtue of the uniqueness of the solution to this Dirichlet problem, we have $u(x) \equiv 0$ in $\Omega$. The theorem is proven.

Let us consider a theorem on the existence of a solution to the problem (24) and (25).

Theorem 6. If coefficients of the operator $L_{n}$ satisfy the condition $\sum_{i=0}^{2^{n}-1}(-1)^{k \otimes i} a_{i} \neq 0$, $k=0, \ldots, 2^{n}-1$, then for functions $f(x) \in C^{\lambda}(\bar{\Omega}), g_{0}(x) \in C^{\lambda+2}(\partial \Omega)$ and $g_{1}(x) \in C^{\lambda+1}(\partial \Omega)$, $0<\lambda<1$, the solution to the problem (24) and (25) exists, is unique, and can be represented as:

$$
u(x)=\sum_{i \equiv\left(i_{n} \ldots i_{1}\right)_{2}=0}^{(1 \ldots 1)_{2}} b_{i} v\left(S_{n}^{i_{n}} \ldots S_{1}^{i_{1}} x\right),
$$


where the function $v(x)$ is a solution to the Dirichlet problem:

$$
\Delta^{2} v(x)=f(x), x \in \Omega ; \quad v(x)=\tilde{g}_{0}(x), \frac{\partial v(x)}{\partial v}=\tilde{g}_{1}(x), x \in \partial \Omega,
$$

with functions:

$$
\tilde{g}_{0}(x)=\sum_{i \equiv\left(i_{n} \ldots i_{1}\right)_{2}=0}^{(1 \ldots 1)_{2}} a_{i} g_{0}\left(S_{n}^{i_{n}} \ldots S_{1}^{i_{1}} x\right), \quad \tilde{g}_{1}(x)=\sum_{i \equiv\left(i_{n} \ldots i_{1}\right)_{2}=0}^{(1 \ldots 1)_{2}} a_{i} g_{1}\left(S_{n}^{i_{n}} \ldots S_{1}^{i_{1}} x\right)
$$

on the boundary, while $b_{i}, i=0, \ldots, 2^{n}-1$ are the coefficients of the first row of the matrix $B_{n}\left(b_{0}, \ldots, b_{2^{n}-1}\right)$ inverse to the matrix $A_{n}=A_{n}\left(a_{0}, \ldots, a_{2^{n}-1}\right)$.

Proof. Let $u(x)$ be a solution to the problem (24) and (25). Consider the function $v(x)=\sum_{i \equiv\left(i_{n} \ldots i_{1}\right)_{2}=0}^{(1 \ldots 1)_{2}} a_{i} u\left(S_{n}^{i_{n}} \ldots S_{1}^{i_{1}} x\right)$. Then, for the function $v(x)$, by virtue of Lemma 7 , we obtain the following boundary value problem:

$$
\begin{gathered}
\Delta^{2} v(x)=f(x), x \in \Omega, \\
\left.\frac{\partial^{j} v(x)}{\partial v^{j}}\right|_{\partial \Omega}=\left.\delta^{j} \sum_{i=0}^{2^{n}-1} a_{k} u\left(S_{n}^{i_{n}} \ldots S_{1}^{i_{1}} x\right)\right|_{\partial \Omega}=\left.\sum_{i=0}^{2^{n}-1} a_{k} I_{S_{n}^{i_{n}} \ldots S_{1}^{i_{1}}} \delta^{j} u(x)\right|_{\partial \Omega} \\
=\sum_{i=0}^{2^{n}-1} a_{k} I_{S_{n}^{i_{n}} \ldots S_{1}^{i_{1}}} g_{j}(x)=\sum_{i=0}^{2^{n}-1} a_{k} g_{j}\left(S_{n}^{i_{n}} \ldots S_{1}^{i_{1}} x\right)=\tilde{g}_{j}(x),
\end{gathered}
$$

where $j=0,1$. If $g_{j}(x) \in C^{2+\lambda-j}(\partial \Omega), j=0,1$, then it is obvious that:

$$
\tilde{g}_{j}(x)=\sum_{i=0}^{2^{n}-1} a_{k} g_{j}\left(S_{n}^{i_{n}} \ldots S_{1}^{i_{1}} x\right) \in C^{2+\lambda-j}(\partial \Omega) .
$$

It is known (see, for example, [39]) that for given functions $f(x)$ and $\tilde{g}_{j}(x)=\sum_{i=0}^{2^{n}-1}$ $a_{k} g_{j}\left(S_{n}^{i_{n}} \ldots S_{1}^{i_{1}} x\right), j=0,1$, a solution to the Dirichlet problem (28) exists and is unique. As in the case of the equality (5) between functions $v(x)$ and $u(x)$, we have the algebraic relation $V(x)=A_{n} U(x)$. If $\sum_{i=0}^{2^{n}-1}(-1)^{k \otimes i} a_{i} \neq 0$, then, by virtue of the equality (26), the unknown function $u(x)$ is uniquely determined through the function $v(x)$. On the contrary, let the function $v(x)$ be a solution to the problem (28). Let us show that the function $u(x)$ defined by Formula (27) satisfies all conditions of the problem (24) and (25). Indeed, if $f(x) \in C^{\lambda}(\bar{\Omega}), g_{j}(x) \in C^{2+\lambda-j}(\partial \Omega), j=0,1$, then we obtain $v(x) \in C^{4}(\Omega) \cap C^{2+\lambda}(\bar{\Omega})$. From here, it follows that $u(x) \in C^{4}(\Omega) \cap C^{2+\lambda}(\bar{\Omega})$. Therefore, according to Lemma 7 , in $\Omega$, we have the equalities:

$$
\begin{gathered}
\Delta^{2} u(x)=\sum_{j=0}^{2^{n}-1} b_{j} \Delta^{2} v\left(S_{n}^{j_{n}} \ldots S_{1}^{j_{1}} x\right)=\sum_{j=0}^{2^{n}-1} b_{j} I_{S_{n}^{j_{n}} \ldots j_{1}^{j_{1}}} \Delta^{2} v(x)=\sum_{j=0}^{2^{n}-1} b_{j} I_{S_{n}^{j_{n}} \ldots S_{1}^{j_{1}}} f(x) \\
=\sum_{j=0}^{2^{n}-1} b_{j} f\left(S_{n}^{j_{n}} \ldots S_{1}^{j_{1}} x\right) .
\end{gathered}
$$


Let us denote $w(x)=\sum_{j=0}^{2^{n}-1} b_{j} f\left(S_{n}^{j_{n}} \ldots S_{1}^{j_{1}} x\right)$ and consider the function $\hat{u}(x)=\sum_{i=0}^{2^{n}-1}$ $a_{i} w\left(S_{n}^{i_{n}} \ldots S_{1}^{i_{1}} x\right)$. Then, for vectors:

$$
\begin{aligned}
W(x) & =\left(w(x), \ldots, w\left(S_{n}^{i_{n}} \ldots S_{1}^{i_{1}} x\right), \ldots, w\left(S_{n}^{1} \ldots S_{1}^{1} x\right)\right)^{T}, \\
F(x) & =\left(f(x), \ldots, f\left(S_{n}^{j_{n}} \ldots S_{1}^{j_{1}} x\right), \ldots, f\left(S_{n}^{1} \ldots S_{1}^{1} x\right)\right)^{T}
\end{aligned}
$$

the equalities $W(x)=B_{n} F(x)$ and $\hat{U}(x)=A_{n} W(x)$ hold, and hence, $A_{n} W(x)=A_{n} B_{n} F(x)$ $=F(x)$. Thus:

$$
\begin{aligned}
\sum_{i \equiv\left(i_{n} \ldots i_{1}\right)_{2}=0}^{(1 \ldots 1)_{2}} a_{i} \Delta^{2} u\left(S_{n}^{i_{n}} \ldots S_{1}^{i_{1}} x\right) \equiv & \sum_{i \equiv\left(i_{n} \ldots i_{1}\right)_{2}=0}^{(1 \ldots 1)_{2}} a_{i} w\left(S_{n}^{i_{n}} \ldots S_{1}^{i_{1}} x\right) \\
& =\left(A_{n} W(x)\right)_{j=0}=\left(A_{n} B_{n} F(x)\right)_{j=0}=F(x)_{j=0}=f(x) .
\end{aligned}
$$

Therefore, the function $u(x)$ satisfies Equation (24).

Let us check the boundary conditions (25) of the studied problem. Taking into account that:

$$
\left(\tilde{G}_{0}(x)\right)_{j=0}=\tilde{g}_{0}(x)=\sum_{i \equiv\left(i_{n} \ldots i_{1}\right)_{2}=0}^{(1 \ldots 1)_{2}} a_{i} g_{0}\left(S_{n}^{i_{n}} \ldots S_{1}^{i_{1}} x\right)=\left(A_{n} G_{0}(x)\right)_{j=0}
$$

for $x \in \partial \Omega$, according to Lemma 7, we obtain:

$$
\begin{aligned}
\frac{\partial^{k} u(x)}{\partial v^{k}}=\delta^{k} u(x)=\sum_{i \equiv\left(i_{n} \ldots i_{1}\right)_{2}=0}^{(1 \ldots 1)_{2}} b_{i} \delta^{k} v\left(S_{n}^{i_{n}} \ldots S_{1}^{i_{1}} x\right)=\left(B_{n} \delta^{k} V(x)\right)_{j=0} \\
=\left(B_{n} \tilde{G}_{k}(x)\right)_{j=0}=\left(B_{n} A_{n} G_{k}(x)\right)_{j=0}=g_{k}(x),
\end{aligned}
$$

where $k=0,1$, and hence, the boundary conditions (25) for the function $u(x)$ are satisfied. The theorem is proven.

\section{The Neumann Problem}

In this section, we first consider the following analogue of the Neumann problem:

$$
\begin{gathered}
L_{n} u(x)=f(x), x \in \Omega, \\
\frac{\partial u(x)}{\partial v}=g_{0}(x), \quad \frac{\partial^{2} u(x)}{\partial v^{2}}=g_{1}(x), x \in \partial \Omega .
\end{gathered}
$$

First, let us consider a property of the solution to the Dirichlet problem for the classical biharmonic equation. Consider the following problem:

$$
\Delta^{2} w(x)=F(x), x \in \Omega ; \quad w(x)=h_{0}(x), \frac{\partial w(x)}{\partial v}=h_{1}(x), x \in \partial \Omega,
$$

where the function $F(x)$ has the form $F(x)=(\delta+4) f(x)$, and functions $\tilde{h}_{0}(x)$ and $\tilde{h}_{1}(x)$ are defined by the equalities:

$$
\tilde{h}_{0}(x)=\sum_{i \equiv\left(i_{n} \ldots i_{1}\right)_{2}=0}^{(1 \ldots 1)_{2}} a_{i} h_{0}\left(S_{n}^{i_{n}} \ldots S_{1}^{i_{1}} x\right), \quad \tilde{h}_{1}(x)=\sum_{i \equiv\left(i_{n} \ldots i_{1}\right)_{2}=0}^{(1 \ldots 1)_{2}} a_{i} h_{1}\left(S_{n}^{i_{n}} \ldots S_{1}^{i_{1}} x\right) .
$$


Let us assume that functions $f(x), h_{0}(x), h_{1}(x)$ are quite smooth. First, we study the problem for the homogeneous equation, i.e., consider the problem:

$$
\Delta^{2} w(x)=0, x \in \Omega ; \quad w(x)=\tilde{h}_{0}(x), \frac{\partial w(x)}{\partial v}=\tilde{h}_{1}(x), x \in \partial \Omega .
$$

It is known (see, for example, [36]) that the solution to the problem (33) can be represented as $w(x)=w_{0}(x)+\left(1-|x|^{2}\right) w_{1}(x)$, where the functions $w_{0}(x), w_{1}(x)$ are solutions to the following problems:

$$
\begin{gathered}
\Delta w_{0}(x)=0, x \in \Omega ; \quad w(x)=\tilde{h}_{0}(x), x \in \partial \Omega . \\
\Delta w_{1}(x)=0, x \in \Omega ; \quad w_{1}(x)=-\frac{1}{2}\left[\tilde{h}_{1}(x)-\frac{\partial w_{0}(x)}{\partial v}\right], x \in \partial \Omega .
\end{gathered}
$$

If we represent the solutions to the problems (34) and (35) in the form of the Poisson integral, then we obtain:

$$
\begin{gathered}
w_{0}(x)=\int_{\partial \Omega} P(x, y) \tilde{h}_{0}(y) d s_{y}=\int_{\partial \Omega} P(x, y)\left[\sum_{i \equiv\left(i_{n} \ldots i_{1}\right)_{2}=0}^{(1 \ldots 1)_{2}} a_{i} h_{0}\left(S_{n}^{i_{n}} \ldots S_{1}^{i_{1}} y\right)\right] d s_{y}, \\
w_{1}(x)=-\frac{1}{2} \int_{\partial \Omega} P(x, y)\left[\sum_{i \equiv\left(i_{n} \ldots i_{1}\right)_{2}=0}^{(1 \ldots 1)_{2}} a_{i} h_{1}\left(S_{n}^{i_{n}} \ldots S_{1}^{i_{1}} y\right)-\frac{\partial w_{0}(y)}{\partial v}\right] d s_{y},
\end{gathered}
$$

where $P(x, y)=\frac{1}{\omega_{l}} \frac{1-|x|^{2}}{|x-y|^{l}}$ is the Poisson kernel of the Dirichlet problem. Further, it is obvious that $P(0, y)=\frac{1}{\omega_{l}}$, and for the harmonic function $w_{0}(x)$ the equality:

$$
\int_{\partial \Omega} \frac{\partial w_{0}(y)}{\partial v} d s_{y}=0
$$

holds. Moreover, in [18] (Lemma 5.1), it was proven that:

$$
\int_{\partial \Omega} g\left(S_{i} y\right) d s_{y}=\int_{\partial \Omega} g(y) d s_{y} .
$$

Then, for the function $w(x)=w_{0}(x)+\left(1-|x|^{2}\right) w_{1}(x)$, we obtain:

$$
\begin{aligned}
& w(0)=w_{0}(0)+w_{1}(0)=\frac{1}{\omega_{l}} \int_{\partial \Omega}\left[\sum_{i \equiv\left(i_{n} \ldots i_{1}\right)_{2}=0}^{(1 \ldots 1)_{2}} a_{i} h_{0}\left(S_{n}^{i_{n}} \ldots S_{1}^{i_{1}} y\right)\right] d s_{y} \\
&-\frac{1}{2 \omega_{l}} \int_{\partial \Omega}\left[\sum_{i \equiv\left(i_{n} \ldots i_{1}\right)_{2}=0}^{(1 \ldots 1)_{2}} a_{i} h_{1}\left(S_{n}^{i_{n}} \ldots S_{1}^{i_{1}} y\right)\right] d s_{y}=\frac{1}{\omega_{l}}\left(\sum_{i=0}^{2^{n}-1} a_{i}\right) \int_{\partial \Omega}\left[h_{0}(y)-\frac{1}{2} h_{1}(y)\right] d s_{y} \\
&=\frac{1}{2 \omega_{l}}\left(\sum_{i=0}^{2^{n}-1} a_{i}\right) \int_{\partial \Omega}\left[2 h_{0}(y)-h_{1}(y)\right] d s_{y} .
\end{aligned}
$$

Thus, if $w(x)$ is a solution to the problem (33), then the equality:

$$
w(0)=\frac{1}{2 \omega_{l}}\left(\sum_{i=0}^{2^{n}-1} a_{i}\right) \int_{\partial \Omega}\left[2 h_{0}(y)-h_{1}(y)\right] d s_{y}
$$

is valid. 
Let us study the problem (32) when the boundary conditions are homogeneous, i.e., consider the problem:

$$
\Delta^{2} w(x)=(\delta+4) f(x), x \in \Omega ; \quad w(x)=0, \frac{\partial w(x)}{\partial v}=0, x \in \partial \Omega .
$$

In [27], it was proven that the solution to the problem (37) satisfies the equality:

$$
w(0)=\frac{1}{4 \omega_{l}} \int_{\Omega}\left(1-|x|^{2}\right) f(x) d x .
$$

Thus, using the equalities (36) and (38) to solve the problem (32), we obtain:

$$
w(0)=\frac{1}{4 \omega_{l}} \int_{\Omega}\left(1-|x|^{2}\right) f(x) d x+\frac{1}{2 \omega_{l}}\left(\sum_{i=0}^{2^{n}-1} a_{i}\right) \int_{\partial \Omega}\left[2 h_{0}(y)-h_{1}(y)\right] d s_{y} .
$$

Hence, it follows that for the condition $w(0)=0$ to be satisfied, it is necessary and sufficient that the equality:

$$
\frac{1}{2} \int_{\Omega}\left(1-|x|^{2}\right) f(x) d x+\left(\sum_{i=0}^{2^{n}-1} a_{i}\right) \int_{\partial \Omega}\left[2 h_{0}(y)-h_{1}(y)\right] d s_{y}=0
$$

be satisfied. Thus, we proved the following assertion.

Lemma 9. If the function $w(x)$ is a solution to the problem (32), then for the condition $w(0)=0$ to be satisfied, it is necessary and sufficient to satisfy the equality (39).

Let us consider an analogue of the Neumann problem (30) and (31). The following assertion is valid.

Theorem 7. Let the coefficients of the operator $L_{n}$ satisfy the condition $\sum_{i=0}^{2^{n}-1}(-1)^{k \otimes i} a_{i} \neq 0$, for $k=0, \ldots, 2^{n}-1$ and $F(x) \in C^{\lambda+1}(\bar{\Omega}), g_{0}(x) \in C^{\lambda+4}(\partial \Omega), g_{1}(x) \in C^{\lambda+3}(\partial \Omega), 0<\lambda<1$. Then, for the solvability of the problem (30) and (31), it is necessary and sufficient that the condition:

$$
\frac{1}{2} \int_{\Omega}\left(1-|x|^{2}\right) f(x) d x=\left(\sum_{i=0}^{2^{n}-1} a_{i}\right) \int_{\partial \Omega}\left[g_{1}(x)-g_{0}(x)\right] d S_{x}
$$

be satisfied. If a solution to the problem exists, it is unique up to a constant term and belongs to the class $C^{\lambda+4}(\bar{\Omega})$.

Proof. Assume that a solution $u(x)$ to the problem (30) and (31) exists, and let $\delta=\sum_{j=1}^{n} x_{j} \frac{\partial}{\partial x_{j}}$. Let us apply the operator $\delta$ to the function $u(x)$ and denote $v(x)=\delta[u](x)$. Note that for $x \in \partial \Omega$, the equalities hold:

$$
\begin{aligned}
& \left.\frac{\partial u(x)}{\partial v}\right|_{\partial \Omega}=\left.\delta[u](x)\right|_{\partial \Omega}=\left.v(x)\right|_{\partial \Omega} ; \\
& \left.\quad \frac{\partial^{2} u(x)}{\partial v^{2}}\right|_{\partial \Omega}=\left.(\delta-1) \delta[u](x)\right|_{\partial \Omega}=\delta[v](x)-\left.v(x)\right|_{\partial \Omega} .
\end{aligned}
$$


Then, taking into account the equality $\Delta^{2} \delta[u](x)=(\delta+4) \Delta^{2} u(x)$, where $x \in \Omega$ and the commutability of the operators $\delta$ and $I_{S_{i}}$, we obtain:

$$
\begin{aligned}
L_{n} v(x)=\sum_{i \equiv\left(i_{n} \ldots i_{1}\right)_{2}=0}^{(1 \ldots 1)_{2}} a_{i} \Delta^{2} I_{S_{n}^{i_{n}} \ldots S_{1}^{i_{1}}} \delta u(x)=(\delta+4) \sum_{i \equiv\left(i_{n} \ldots i_{1}\right)_{2}=0}^{(1 \ldots 1)_{2}} a_{i} \Delta^{2} u\left(S_{n}^{i_{n}} \ldots S_{1}^{i_{1}} x\right) \\
=(\delta+4) L_{n} u(x)=(\delta+4) f(x) \equiv F(x) .
\end{aligned}
$$

From the boundary conditions of the problem (30) and (31), taking into account the equalities (41), we obtain:

$$
\left.v(x)\right|_{\partial \Omega}=g_{0}(x) ;\left.\frac{\partial v(x)}{\partial v}\right|_{\partial \Omega}=g_{1}(x)+g_{0}(x) .
$$

Thus, if $u(x)$ is a solution to the problem (30) and (31), then for the function $v(x)=\delta[u](x)$, we obtain the following Dirichlet problem:

$$
L_{n} v(x)=(\delta+4) f(x) \equiv F(x), \quad x \in \Omega ;\left.v(x)\right|_{\partial \Omega}=g_{0}(x),\left.\frac{\partial v(x)}{\partial v}\right|_{\partial \Omega}=g_{1}(x)+g_{0}(x) .
$$

Moreover, from the equality $v(x)=\delta[u](x)$, it follows that the solution to this problem must satisfy the condition $v(0)=0$. Let us find out when this condition is fulfilled. We introduce the notation:

$$
w(x)=\sum_{i \equiv\left(i_{n} \ldots i_{1}\right)_{2}=0}^{(1 \ldots 1)_{2}} a_{i} v\left(S_{n}^{i_{n}} \ldots S_{1}^{i_{1}} x\right) .
$$

If $v(x)$ is a solution to the problem (42), then the function $w(x)$ from (43) satisfies the conditions of the problem (32) with the functions:

$$
\tilde{h}_{0}(x)=\sum_{i \equiv\left(i_{n} \ldots i_{1}\right)_{2}=0}^{(1 \ldots 1)_{2}} a_{i} g_{0}\left(S_{n}^{i_{n}} \ldots S_{1}^{i_{1}} x\right), \quad \tilde{h}_{1}(x)=\tilde{h}_{0}(x)+\sum_{i \equiv\left(i_{n} \ldots i_{1}\right)_{2}=0}^{(1 \ldots 1)_{2}} a_{i} g_{1}\left(S_{n}^{i_{n}} \ldots S_{1}^{i_{1}} x\right) .
$$

From (43), it follows that:

$$
w(0)=\sum_{i \equiv\left(i_{n} \ldots i_{1}\right)_{2}=0}^{(1 \ldots 1)_{2}} a_{i} v(0)=\left(\sum_{i \equiv\left(i_{n} \ldots i_{1}\right)_{2}=0}^{(1 \ldots 1)_{2}} a_{i}\right) v(0) .
$$

Then, under the condition $\underset{i \equiv\left(i_{n} \ldots i_{1}\right)_{2}=0}{\left.\sum_{1} \ldots\right)_{2}} a_{i} \neq 0$, the equality $v(0)=0$ is satisfied if and only if the condition $w(0)=0$ is satisfied. In turn, by the assertion of Lemma 9, for the equality $w(0)=0$ to hold, it is necessary and sufficient to satisfy the condition (39). As in our case:

$$
2 h_{0}(y)-h_{1}(y)=2 g_{0}(x)-\left[g_{1}(x)+g_{0}(x)\right]=g_{0}(x)-g_{1}(x),
$$

then the condition for the solvability of the problem (30) and (31) can be rewritten as (40). Thus, the necessity of fulfilling the condition (40) for the existence of a solution to the problem (30) and (31) is proven.

Let us show that the fulfillment of the condition (40) is also sufficient for the existence of a solution to the problem (30) and (31). To do this, consider in $\Omega$ the following Neumann problem with respect to the function $z(x)$ :

$$
\Delta^{2} z(x)=F(x), x \in \Omega,
$$




$$
\begin{gathered}
\left.z(x)\right|_{\partial \Omega}=\sum_{i \equiv\left(i_{n} \ldots i_{1}\right)_{2}=0}^{(1 \ldots 1)_{2}} a_{i} g_{0}\left(S_{n}^{i_{n}} \ldots S_{1}^{i_{1}} x\right) \equiv \tilde{h}_{0}(x), \\
\left.\frac{\partial z(x)}{\partial v}\right|_{\partial \Omega}=\sum_{i \equiv\left(i_{n} \ldots i_{1}\right)_{2}=0}^{(1 \ldots 1)_{2}} a_{i} g_{1}\left(S_{n}^{i_{n}} \ldots S_{1}^{i_{1}} x\right) \equiv \tilde{h}_{1}(x) .
\end{gathered}
$$

It is known (see, for example, [36]) that the solvability condition of this problem can be written as:

$$
\frac{1}{2} \int_{\Omega}\left(1-|x|^{2}\right) F(x) d x=\int_{\partial \Omega}\left[\tilde{h}_{1}(x)-\tilde{h}_{0}(x)\right] d S_{x} .
$$

By virtue of [18] (Lemma 5.1), $\int_{\partial \Omega} g\left(s_{i} x\right) d s_{x}=\int_{\partial \Omega} g(x) d s_{x}$, whence it follows that:

$$
\sum_{i \equiv\left(i_{n} \ldots i_{1}\right)_{2}=0}^{(1 \ldots 1)_{2}} a_{i} \int_{\partial \Omega} g_{0}\left(S_{n}^{i_{n}} \ldots S_{1}^{i_{1}} x\right) d s_{x}=\left(\sum_{i=0}^{2^{n}-1} a_{i}\right) \int_{\partial \Omega} g(x) d s_{x}
$$

and therefore, the condition (37) can be rewritten in the form of (40). If this condition is satisfied, then a solution to the problem (44) and (45) exists and is unique up to a constant term.

Further, as in the case of the Dirichlet problem, the solution to the Neumann problem (30) and (31) can be found by the formula:

$$
u(x)=\sum_{i \equiv\left(i_{n} \ldots i_{1}\right)_{2}=0}^{(1 \ldots 1)_{2}} b_{i} v\left(S_{n}^{i_{n}} \ldots S_{1}^{i_{1}} x\right) .
$$

Indeed, if we consider the vector $Z=\left(z(x), \ldots, z\left(S_{n}^{j_{n}} \ldots S_{1}^{j_{1}} x\right), \ldots, z\left(S_{n}^{1} \ldots S_{1}^{1} x\right)\right)^{T}$, then from the vector equality $U=A_{n}^{-1} Z$, if the conditions of the theorem are satisfied, we can determine the vector $U(x)=\left(u(x), \ldots, u\left(S_{n}^{i_{n}} \ldots S_{1}^{i_{1}} x\right), \ldots, u\left(S_{n}^{1} \ldots S_{1}^{1} x\right)\right)^{T}$. As $A_{n} U=Z$, then the function $u(x)$ is uniquely determined through the function $z(x)$ from (44) and (45) by Formula (47). In a similar way, as in the case of the Dirichlet problem, it can be shown that the function (47) satisfies all conditions of the problem (30) and (31). The theorem is proven.

Further, we define the solvability condition for the problem $H$ in the case $\alpha=1, \mu=0$. In this case, the boundary conditions are written as:

$$
\frac{\partial u(x)}{\partial v}=g_{0}(x), \quad \frac{\partial^{2} u(x)}{\partial v^{2}}+\frac{\partial u(x)}{\partial v}=g_{1}(x), x \in \partial \Omega .
$$

The second condition from (48), taking into account the first condition, can be rewritten in the form:

$$
\frac{\partial^{2} u(x)}{\partial v^{2}}=g_{1}(x)-g_{0}(x) .
$$

Then, the problem under consideration is equivalent to the problem (30) and (31) with the following boundary conditions:

$$
\frac{\partial u(x)}{\partial v}=g_{0}(x), \quad \frac{\partial^{2} u(x)}{\partial v^{2}}=g_{1}(x)-g_{0}(x), x \in \partial \Omega .
$$

Therefore, Theorem 7 implies the following assertion. 
Corollary 7. Let $\alpha=1, \mu=0$, the coefficients of the operator $L_{n}$, satisfy the condition $\sum_{i=0}^{2^{n}-1}$ $(-1)^{k \otimes i} a_{i} \neq 0$, for $k=0, \ldots, 2^{n}-1$ and $0<\lambda<1, f(x) \in C^{\lambda+1}(\bar{\Omega}), g_{0}(x) \in C^{\lambda+4}(\partial \Omega)$, $g_{1}(x) \in C^{\lambda+3}(\partial \Omega)$. Then, for the solvability of the problem $H$, it is necessary and sufficient that the condition:

$$
\frac{1}{2} \int_{\Omega}\left(1-|x|^{2}\right) f(x) d x=\left(\sum_{i=0}^{2^{n}-1} a_{i}\right) \int_{\partial \Omega}\left[g_{1}(x)-2 g_{0}(x)\right] d S_{x}
$$

be satisfied. If a solution to the problem exists, it is unique up to a constant term and belongs to the class $C^{\lambda+4}(\bar{\Omega})$.

\section{The General Case of the Main Problem}

In this section, we consider the general case of the problem $H$. The following assertion is true.

Theorem 8. Let $0<\alpha \leq 1, \mu \geq 0$, the coefficients of the operator $L_{n}$, satisfy the condition $\sum_{i=0}^{2^{n}-1}(-1)^{k \otimes i} a_{i} \neq 0$, for $k=0, \ldots, 2^{n}-1$ and $f(x) \in C^{\lambda+1}(\bar{\Omega}), g_{0}(x) \in C^{\lambda+4}(\partial \Omega), g_{1}(x) \in$ $C^{\lambda+3}(\partial \Omega), 0<\lambda<1$. Then:

(1) If $\mu>0$, then a solution to the problem $H$ exists, is unique, belongs to the class $C^{\lambda+4}(\bar{\Omega})$, and can be represented in the form:

$$
u(x)=J_{\mu}^{\alpha}[v](x),
$$

where $v(x)$ is a solution of the following Dirichlet problem:

$$
L_{n} v(x)=D_{\mu+4}^{\alpha}[f](x), x \in \Omega ; \quad v(x)=g_{0}(x), \frac{\partial v(x)}{\partial v}=g_{1}(x), x \in \partial \Omega
$$

(2) If $\mu=0$, then for solvability of the problem $H$, it is necessary and sufficient that the condition:

$$
\frac{1}{2} \int_{\Omega}\left(1-|x|^{2}\right) f_{1-\alpha}(x) d x=\left(\sum_{k=1}^{l} a_{k}\right) \int_{\partial \Omega}\left[g_{1}(x)-2 g_{0}(x)\right] d S_{x}
$$

be satisfied, where $f_{1-\alpha}(x)$ is defined by the equality $f_{1-\alpha}(x)=J_{4}^{1-\alpha}[f](x)$.

If a solution to the problem exists, it is unique up to a constant term, belongs to the class $C^{\lambda+4}(\bar{\Omega})$, and can be represented as:

$$
u(x)=C+J_{0}^{\alpha}[v](x), C=\text { const }
$$

where $v(x)$ is a solution to the problem (51) for $\mu=0$.

Proof. Let the function $u(x)$ be a solution to the problem $H$. We apply the operator $D_{\mu}^{\alpha}$ to this function and denote $v(x)=D_{\mu}^{\alpha}[u](x)$. Let us apply the operator $\Delta^{2}$ to this function. Then, by virtue of the equality (20), $\Delta^{2} v(x)=D_{\mu+4}^{\alpha}\left[\Delta^{2} u\right](x)$. From here, it follows that for any $k=0, \ldots, 2^{n}-1, \Delta^{2} v\left(S_{n}^{i_{n}} \ldots S_{1}^{i_{1}} x\right)=D_{\mu+4}^{\alpha}\left[\Delta^{2} u\right]\left(S_{n}^{i_{n}} \ldots S_{1}^{i_{1}} x\right)$. Hence,

$$
L_{n} v(x)=\sum_{i=0}^{2^{n}-1} a_{i} \Delta^{2} D_{\mu}^{\alpha} u\left(S_{n}^{i_{n}} \ldots S_{1}^{i_{1}} x\right)=D_{\mu+4}^{\alpha}\left[\sum_{i=0}^{2^{n}-1} a_{i} \Delta^{2} u\left(S_{n}^{i_{n}} \ldots S_{1}^{i_{1}} x\right)\right]=D_{\mu+4}^{\alpha}[f](x),
$$

where $x \in \Omega$. 
In [27], it was proven that the function $D_{\mu}^{\alpha+1}[u](x)$ can be represented as $D_{\mu}^{\alpha+1}[u](x)=$ $\delta\left[D_{\mu}^{\alpha+1}[u]\right](x)$. Then, from the boundary conditions (2) and (3), it follows that:

$$
\left.v(x)\right|_{\partial \Omega}=\left.D_{\mu}^{\alpha}[u](x)\right|_{\partial \Omega}=g_{0}(x),\left.\quad \frac{\partial v(x)}{\partial v}\right|_{\partial \Omega}=\left.\delta\left[D_{\mu}^{\alpha}[u]\right](x)\right|_{\partial \Omega}=g_{1}(x) .
$$

Thus, if $u(x)$ is a solution to the problem $H$, then for the function $v(x)=D_{\mu}^{\alpha}[u](x)$, we obtain the Dirichlet problem (51). If $f(x) \in C^{\lambda+1}(\bar{\Omega})$, then by virtue of Lemma 2, $D_{\mu+4}^{\alpha}[f](x) \in C^{\lambda}(\bar{\Omega})$. Moreover, by the condition of the theorem $g_{0}(x) \in C^{\lambda+4}(\partial \Omega)$, $g_{1}(x) \in C^{\lambda+3}(\partial \Omega)$. Then, by virtue of the assertion of Theorem 6 , a solution to the problem (51) exists, is unique, and belongs to the class $C^{\lambda+4}(\bar{\Omega})$. If we apply the operator $J_{\mu}^{\alpha}$ to both sides of the equality $v(x)=D_{\mu}^{\alpha}[u](x)$, then by virtue of Lemma 3 in the case $\mu>0$, we obtain $u(x)=J_{\mu}^{\alpha}[v](x)$, that is, if a solution to the problem $H$ exists, then the representation (50) is valid for it.

Let, on the contrary, the function $v(x)$ be a solution to the problem (51). Let us show that the function $u(x)=J_{\mu}^{\alpha}[v](x)$ satisfies all the conditions of the problem $H$. Indeed, if we apply the operator $L_{n}$ to this function, then we obtain:

$$
L_{n} u(x)=L_{n}\left[J_{\mu}^{\alpha}[v]\right](x)=J_{\mu+4}^{\alpha}\left[L_{n} v\right](x)=J_{\mu+4}^{\alpha}\left[D_{\mu+4}^{\alpha}[f]\right](x)=f(x), x \in \Omega,
$$

i.e., the function $u(x)=J_{\mu}^{\alpha}[v](x)$ satisfies Equation (1). Let us check the fulfillment of the boundary conditions (2) and (3). By the assertion of Lemma 4, namely by virtue of the equality (19) in the case $\mu>0$, the equalities:

$$
\begin{gathered}
\left.D_{\mu}^{\alpha}[u](x)\right|_{\partial \Omega}=\left.D_{\mu}^{\alpha}\left[J_{\mu}^{\alpha}[v]\right](x)\right|_{\partial \Omega}=\left.v(x)\right|_{\partial \Omega}=g_{0}(x), \\
\left.D_{\mu}^{\alpha}[u](x)\right|_{\partial \Omega}=\left.r \frac{\partial}{\partial r} D_{\mu}^{\alpha}\left[J_{\mu}^{\alpha}[v]\right](x)\right|_{\partial \Omega}=\left.r \frac{\partial v(x)}{\partial r}\right|_{\partial \Omega}=\left.\frac{\partial v(x)}{\partial v}\right|_{\partial \Omega}=g_{1}(x),
\end{gathered}
$$

are satisfied, i.e., the boundary conditions are also satisfied.

It remains to investigate the case $\mu=0$. In this case, the function $v(x)$-a solution to the problem (51) - must satisfy the additional condition $v(0)=0$.

As the function $F(x)=D_{\mu+4}^{\alpha}[f](x)$ by virtue of Lemma 6 is represented as $F(x)=$ $(\delta+4) f_{1-\alpha}(x), f_{1-\alpha}(x)=J_{4}^{1-\alpha}[f](x)$, then, by Lemma 9 , for the equality $v(0)=0$ to hold, it is necessary and sufficient that condition (39) be satisfied. In our case, $2 h_{0}(y)-h_{1}(y)=$ $2 g_{0}(x)-g_{1}(x)$, and therefore, the condition (39) can be rewritten in the form of (52). Thus, the necessity of the fulfillment of the condition (52) for the existence of a solution to the problem $H$ is proven. The other part of the theorem can be proven in the same way as in the case $\mu>0$. The theorem is proven.

Remark 3. If $\alpha=1$ and $\mu=0$, then $f_{0}(x)=J_{4}^{0}[f](x) \equiv f(x)$, and the solvability condition (52) coincides with the condition (49).

\section{Conclusions}

Summarizing the investigation carried out, we note that with the help of transformations of the involution-type, a nonlocal analogue of the biharmonic operator was introduced. Then, for the corresponding biharmonic equation with multiple involutions, the solvability of the boundary value problems with a fractional-order boundary operator having a derivative of the Hadamard-type was investigated. Theorems on the existence and uniqueness of solutions to the problems under consideration were proven. Exact solvability conditions were found depending on the order of the boundary operators. In this work, we extended the correct statement of the boundary value problems for some class of nonlocal partial differential equations. In the future, we plan to investigate spectral questions for operators with multiple involutions. 
Author Contributions: : Conceptualization, B.T., V.K. and M.M.; investigation, B.T., V.K. and M.M.; writing-original draft preparation, B.T., V.K. and M.M.; writing-review and editing, B.T., V.K. and M.M. All authors read and agreed to the published version of the manuscript.

Funding: The research of the first and third authors was supported by the grant of the Committee of Sciences, Ministry of Education and Science of the Republic of Kazakhstan, Project AP09259074. The second author was supported by Act 211 of the Government of the Russian Federation, Contract No. 02.A03.21.0011.

Institutional Review Board Statement: Not applicable.

Informed Consent Statement: Not applicable.

Data Availability Statement: All the data are present within the manuscript.

Conflicts of Interest: The authors declare no conflict of interest.

\section{References}

1. Nahushev, A.M. Equations of Mathematical Biology; Nauka: Moscow, Russia, 1995. (In Russian)

2. Cabada, A.; Tojo, F.A.F. Differential Equations with Involutions; Atlantis Press: New York, NY, USA, 2015.

3. Karapetiants, N.; Samko, S. Equations with Involutive Operators; Birkhäuser: Boston, MA, USA, 2001.

4. Litvinchuk, G.S. Solvability Theory of Boundary Value Problems and Singular Integral Equations with Shift; Kluwer Academic Publishers: Boston, MA, USA, 2000.

5. Al-Salti, N.; Kirane, M.; Torebek, B.T. On a class of inverse problems for a heat equation with involution perturbation. Hacet. J. Math. Stat. 2019, 48, 669-681. [CrossRef]

6. Al-Salti, N.; Kerbal, S.; Kirane, M. Initial-boundary value problems for a time-fractional differential equation with involution perturbation. Math. Model. Nat. Phenom. 2019, 14,1-15. [CrossRef]

7. Andreev, A.A. Analogs of classical boundary value problems for a second-order differential equation with deviating argument. Differ. Equ. 2004, 40, 1192-1194. [CrossRef]

8. Ashyralyev, A.; Sarsenbi, A.M. Well-posedness of an elliptic equation with involution. Electron. J. Differ. Equ. 2015, $284,1-8$.

9. Ashyralyev, A.; Sarsenbi, A.M. Well-posedness of a parabolic equation with involution. Numer. Funct. Anal. Optim. 2017, 38, 1295-1304. [CrossRef]

10. Baskakov, A.G.; Uskova, N.B. Fourier method for first order differential equations with involution and groups of operators. Ufa Math. J. 2018, 10, 11-34. [CrossRef]

11. Baskakov, A.G.; Krishtal, I.A.; Uskova, N.B. On the spectral analysis of a differential operator with an involution and general boundary conditions. Eurasian Math. J. 2020, 11, 30-39. [CrossRef]

12. Burlutskaya, M. Some properties of functional-differential operators with involution $v(x)=1-x$ and their applications. Russ. Math. 2021, 5, 89-97.

13. Burlutskaya, M. Mixed problem for a first-order partial differential equation with involution and periodic boundary conditions.Comput. Math. Math. Phys. 2014, 54,1-10. [CrossRef]

14. Kirane, M.; Al-Salti, N. Inverse problems for a nonlocal wave equation with an involution perturbation. J. Nonlinear Sci. Appl. 2016, 9, 1243-1251. [CrossRef]

15. Ruzhansky, M.; Tokmagambetov, N.; Torebek, B.T. On a non-local problem for a multi-term fractional diffusion-wave equation. Fract. Calc. Appl. Anal. 2020, 23, 324-355. [CrossRef]

16. Torebek, B.T.; Tapdigoglu, R. Some inverse problems for the nonlocal heat equation with Caputo fractional derivative. Math. Methods Appl. Sci. 2017, 40, 6468-6479. [CrossRef]

17. Przeworska-Rolewicz, D. Some boundary value problems with transformed argument. Comment. Math. 1974, 17, 451-457.

18. Karachik, V.V.; Turmetov, B. On solvability of some nonlocal boundary value problems for biharmonic equation. Math. Slovaca 2020, 70, 329-342. [CrossRef]

19. Karachik, V.V.; Turmetov, B. Solvability of one nonlocal Dirichlet problem for the Poisson equation. Novi Sad J. Math. 2020, 50, 67-88.

20. Karachik, V.V.; Sarsenbi, A.M.; Turmetov, B. On the solvability of the main boundary value problems for a nonlocal Poisson equation. Turk. J. Math. 2019, 43, 1604-1625. [CrossRef]

21. Yarka, U.; Fedushko, S.; Vesely, P. The Dirichlet Problem for the Perturbed Elliptic Equation. Mathematics 2020, 8, 2108. [CrossRef]

22. Ashurov, R.; Fayziev, Y. On some boundary value problems for equations with boundary operators of fractional order. Int. J. Appl. Math. 2021, 34, 283-295. [CrossRef]

23. Vasylyeva, N. Local Solvability of a Linear System with a Fractional Derivative in Time in a Boundary Condition. Fract. Calc. Appl. Anal. 2015, 18, 982-1005. [CrossRef]

24. Gorenflo, R.; Luchko, Y.F.; Umarov, S.R. On some boundary value problems for pseudo-differential equations with boundary operators of fractional order. Fract. Calc. Appl. Anal. 2000, 3, 453-468. [CrossRef] 
25. Kirane, M.; Torebek, B.T. On a nonlocal problem for the Laplace equation in the unit ball with fractional boundary conditions. Math. Methods Appl. Sci. 2016, 39, 1121-1128. [CrossRef]

26. Krasnoschok, M.; Vasylyeva, N. On a nonclassical fractional boundary-value problem for the Laplace operator. J. Differ. Equ. 2014, 257, 1814-1839. [CrossRef]

27. Turmetov, B. On the solvability of some boundary value problems for the inhomogeneous polyharmonic equation with boundary operators of the Hadamard type. Differ. Equ. 2017, 53, 333-344.

28. Turmetov, B.; Nazarova, K. On fractional analogs of Dirichlet and Neumann problems for the Laplace equation. Mediterr. J. Math. 2019, 16, 1-17.

29. Turmetov, B.; Nazarova, K. On a generalization of the Neumann problem for the Laplace equation. Math. Nachrichten 2020, 293, 169-177. [CrossRef]

30. Umarov, S.R. Some boundary value problems for elliptic equations with a boundary operator of fractional order. Dokl. Akad. Nauk. 1993, 333, 708-710. (In Russian) [CrossRef]

31. Veliev, T.M.; Ivakhnychenko, M.T.; Ahmedov, T. Fractional boundary conditions in plane waves diffraction on a strip. Prog. Electromagn. Res. 2008, 79, 443-462. [CrossRef]

32. Ivakhnychenko, M.; Veliev, E.; Akhmedov, T. Fractional operators approach in electromagnetic wave reflection problems. J. Electromagn. Waves Appl. 2007, 21, 1787-1802.

33. Tabatadze, V.; Karaçuha, K.; Veliev, E. The solution of the plane wave diffraction problem by two strips with different fractional boundary conditions. J. Electromagn. Waves Appl. 2020, 34, 881-893.

34. Tabatdze, V.; Karaçuha, K.; Veliev, E.; Karaçuha, E. Diffraction of the electromagnetic plane waves by double half-plane with fractional boundary conditions. Prog. Electromagn. Res. M 2021, 101, 207-218.

35. Kilbas, A.A.; Srivastava, H.M.; Trujillo, J.J. Theory and Applications of Fractional Differential Equations; Elsevier: Amsterdam, The Netherlands, 2006.

36. Bitsadze, A.V. On polyharmonic functions. Dokl. Akad. Nauk SSSR 1987, 294, 521-525. (In Russian)

37. Karachik, V.V. Class of Neumann-type problems for the polyharmonic equation in a ball. Comput. Math. Math. Phys. 2020, 60, 144-162.

38. Karachik, V.V. Generalized Third Boundary Value Problem for the Biharmonic Equation. Differ. Equ. 2017, 53, 756-765.

39. Agmon, S.; Douglis, A.; Nirenberg, L. Estimates near the boundary for solutions of elliptic partial differential equations satisfying general boundary conditions. I. Commun. Pure Appl. Math. 1959, 12, 623-727. 